Note du comité de rédaction : Le problème de l'effet des faibles doses est d'actualité : notre revue vient de recevoir une contribution du Professeur Maurice Tubiana consacrée à cette question. Le comité de rédaction a décidé de publier rapidement cette étude, pour apporter à nos lecteurs une information de première main, donnant un point de vue précis sur la question. Nous souhaitons que Radioprotection amorce ainsi une discussion générale afin que s'établisse un débat permettant aux diverses tendances de s'exprimer en toute liberté.

D. BLANC

Article

\title{
Effets cancérogènes des faibles doses du rayonnement ionisant*
}

\author{
M. TUBIANA**
}

(Manuscrit reçu le 8 décembre 1995, accepté le 27 février 1996)

RÉSUMÉ Les enquêtes épidémiologiques, même celles portant sur plusieurs dizaines de milliers de sujets (comme chez les survivants d'Hiroshima et Nagasaki) ne décèlent pas d'effet cancérogène chez l'enfant ou l'homme adulte pour des doses inférieures à $200 \mathrm{mSv}$ en irradiation aiguë ou $500 \mathrm{mSv}$ en irradiation à faible débit. Alors que la dose due au rayonnement naturel varie entre 1,5 et $6 \mathrm{mSv}$ et même parfois davantage, les enquêtes n'ont pas pu déceler de variation dans la fréquence des cancers en fonction de cette irradiation naturelle. Ces résultats ne permettent pas d'exclure l'hypothèse d'un effet cancérogène des faibles doses mais permettent de fixer la limite supérieure de l'effet éventuel dans cette gamme de dose. Pour estimer celui-ci on se fonde donc généralement sur les effets observés à dose élevée en effectuant une extrapolation vers les doses faibles. En 1990 le CIPR a révisé à la hausse l'évaluation de l'effet cancérogène à dose élevée, principalement à cause de l'introduction d'un modèle mathématique destiné à estimer l'effet cancérogène jusqu'à la fin de l'existence des personnes irradiées. Le modèle utilisé admet que l'irradiation introduit un facteur multiplicatif constant jusqu'au décès. Des résultats récents montrent qu'en réalité cet effet diminue avec le temps donc que cette hypothèse entraîne une surestimation du risque. Pour estimer les effets à dose faible le CIPR procède à une extrapolation linéaire assortie d'un facteur de réduction égal à deux. L'intérêt de cette méthode est d'indiquer la limite supérieure du risque mais elle peut surestimer notablement celui-ci à faible dose. En effet cette proportionnalité de l'effet avec la dose même pour les doses et les débits de dose les plus faibles implique deux postulats : 1) l'efficacité de la réparation des lésions de l'ADN dans les cellules ne varie pas avec la dose et le débit de dose. 2) la traversée d'une seule cellule par une seule particule entraîne un risque de transformation cancérogène. Si ces hypothèses étaient exactes le débit de dose ne devrait pas avoir d'influence pour les doses faibles mais ce n'est pas ce que montrent les données expérimentales. De plus les récents progrès faits dans l'étude des systèmes de réparation de l'ADN montrent qu'après de fortes doses ceux-ci peuvent être saturés et des systèmes de réparation moins fidèles être mis en euvre. D'autre part la cancérogenèse est un phénomène très complexe qui requiert plusieurs lésions indépendantes du génome qu'une seulè particule est incapable de provoquer. D'ailleurs pour les particules

* Texte corrigé d'une conférence faite à Montpellier en juin 1995.

** Directeur honoraire de l'Institut Gustave Roussy, Membre de l'Académie des Sciences et de l'Académie de Médecine, Centre Antoine Béclère, Faculté de Médecine, 45, rue des SaintsPères, 75006 Paris, France. 
déterminant la plus forte densité d'ionisation le long de la trajectoire de la particule (particules alpha) qui sont celles pour lesquelles il serait concevable qu'une seule particule détermine plusieurs lésions, aucun effet cancérogène n'est observé pour des doses faibles en opposition avec les conclusions de ce modèle. Ainsi l'effet cancérogène des faibles doses pourrait être notablement inférieur à celui qui avait été estimé en 1991. L'avancée rapide des connaissances tant pour les mécanismes fondamentaux que pour les enquêtes épidémiologiques fait espérer que l'estimation du risque des faibles doses pourra dans les prochaines années être de plus en plus précise. Il est d'autant plus légitime de ne pas se hâter pour modifier les normes que moins de $1 \%$ des travailleurs reçoivent actuellement une dose supérieure à la limite de dose annuelle actuellement proposée et que dans l'enquête du CIRC moins de 1 pour 1000 des 90000 travailleurs de l'industrie nucléaire étudiés n'avaient reçu au cours de leur vie professionnelle une dose avoisinant $1 \mathrm{~Sv}$, dose limite-vie proposée à la fois dans les rapports de l'Académie des Sciences et du CIPR.

ABSTRACT Epidemiological studies, even those performed on tens of thousands of subjects (such as Hiroshima and Nagasaki survivors), have not produced evidence of a carcinogenic effect in children or adult individuals for doses lower than $200 \mathrm{mSv}$ in acute irradiation or $500 \mathrm{mSv}$ in low dose-rate irradiation. Even though doses due to natural irradiation vary between 1.5 and $6 \mathrm{mSv}$ per year, and sometimes more, studies have been unable to detect variations in the incidence of cancers as a function of this background irradiation. These results do not permit the exclusion of the hypothesis of the carcinogenic effect of low doses, but they show that the value of the eventual carcinogenic effect in this range of dose should be small and within the limit of the error bars of these studies. Estimations usually are made based on the effects observed at high doses which are extrapolated to low doses. In 1990 the ICRP revised upward the evaluation of the carcinogenic effect at high doses, principally because of the introduction of a mathematical model aimed at estimating the carcinogenic. effect until the end of the irradiated individuals' existence. This model assumes that irradiation introduces a constant multiplying factor until the end of existence. Recent results have shown that in fact the effect decreases over time and that therefore this hypothesis leads to an overestimation of risk. To estimate low dose effects, the ICRP uses a linear extrapolation associated with a dose-rate reduction factor which has been chosen equal to two. This method indicates the highest limit of risk, but it may overestimate the risk at low doses. This proportionality of the effect with the dose, even for the lowest doses and dose-rates, which is implicitly based on the linear quadratic model leads to two assumptions: 1) the efficacy of DNA repair in cells does not vary with the dose and the dose-rate; 2) a single physical event in a single cell crossed by a single particle may induce carcinogenesis. If these assumptions were correct, the dose-rate should not have an influence within the low doses range; however, this is not upheld by experimental data. Moreover, the recent progress made in the study of DNA repair systems has shown that after high doses these systems can become saturated and that systems which are more error prone are activated. Furthermore, carcinogenesis is a very complex phenomenon that requires several independent lesions of the genome that a single particle is incapable of inducing. For particles determining the greatest density of ionization along the path of particles (alpha particles), which are those for which it would be conceivable that one particle determines several irreparable lesions, no carcinogenic effect has been observed for low doses in opposition with the assumptions of this model. Therefore the carcinogenic effect of low doses could be markedly lower than what was estimated in 1991. Rapid advances in the understanding of fundamental mechanisms and epidemiological studies give rise to hopes that the estimation of risk of low doses will become more and more precise in the next few years. There is no urgency for introducing new regulations since only $1 \%$ of workers currently receive a dose higher than the annual dose limit presently proposed. Moreover, in 


\begin{abstract}
a recent study by IARC, less than 1 for 1,000 of the 90,000 nuclear industry workers studied had received during their professional life a dose approaching $1 \mathrm{~Sv}$, which is the life dose limit proposed in both the reports of 1989 and 1995 of the French Academy of Science and the ICRP.
\end{abstract}

Depuis 1989 les nouvelles normes de radioprotection proposées par l'ICRP (International Commission on Radiological Protection) ont suscité des débats autour des risques des faibles doses du rayonnement.

En 1989 la diffusion par l'ICRP de ses projets avait amené le Ministère de la Recherche à demander à l'Académie des Sciences son avis sur cette question. La conclusion de son rapport, en novembre 1989, avait été : "Au total, considérant que les normes actuelles paraissent déjà prudentes, qu'il n'existe aucune raison scientifique de les remettre en cause, que l'on pourrait en quelques années compléter les données sur les effets des faibles doses, il apparaît qu'un effort prioritaire devrait être effectué pour développer les recherches épidémiologiques sur les effets des faibles doses et qu'il serait préférable d'attendre le résultat de ces études pour procéder à une révision des normes" (Académie des Sciences, 1989).

Tout naturellement, cinq ans plus tard, à l'automne 1994, le Ministre de la Santé saisit à nouveau l'Académie des Sciences afin de savoir si à la lumière de la dernière publication de l'ICRP (1991) et des plus récents travaux, son point de vue avait changé. Le rapport qui a été remis au gouvernement au printemps 1995 et publié en novembre (Académie des Sciences, 1995) conclut qu'il n'existe pas de faits scientifiques indiscutables et récents apportant un argument en faveur d'un abaissement des normes en vigueur en France pour les travailleurs ou le public. Signalons cependant qu'une petite minorité du groupe a estimé qu'il ne lui paraissait pas souhaitable que la France se dissocie des positions des autres pays.

Avant d'analyser les données scientifiques sur lesquelles ces conclusions sont fondées, l'auteur de cet article voudrait faire deux remarques : 1) bien qu'il ait participé à la rédaction de ces deux rapports les commentaires ci-dessous n'engagent que lui, 2) la fixation et la révision de normes de protection implique outre la prise en compte des données scientifiques un jugement de valeur sur la quantité de risque acceptable. Aucune activité humaine n'est exempte de risque et déterminer la quantité de risque acceptable fait intervenir des notions éthiques et sociales. Dans ses rapports précédents l'ICRP avait indiqué que pour le public la quantité de risque devait être comparable à celle acceptée dans des activités usuelles, par exemple les transports en commun, tandis que pour le travailleur on devait se rapporter à des activités industrielles classiques ayant un risque professionnel faible. Ces repères, malgré leur grande valeur, comportent toutefois une imprécision qui explique que certains auteurs puissent dire que le risque ayant diminué tant dans les activités industrielles que dans les transports il faut abaisser les normes, alors que d'autres auteurs (Académie des Sciences, 1989; Chau, 1987) soulignent que les normes de radioprotection anciennes $(50 \mathrm{mSv} / \mathrm{an}$ pour les travailleurs, $5 \mathrm{mSv} / \mathrm{an}$ pour le public, ICRP, 1977), donnaient déjà une marge de sécurité importante par rapport à ces activités. 


\section{Position du problème : l'évolution du coefficient de risque cancérogène}

Le fait essentiel du rapport 60 de l'ICRP (1991) a été la réévaluation du risque cancérogène à faible dose. Le tableau I indique l'évolution du coefficient de risque; comme on le voit il est du à deux facteurs : l'augmentation importante du risque à dose élevée, le choix pour le facteur de réduction pour faible dose-faible débit de la valeur la plus péjorative sur la fourchette précédemment proposée par le Comité National américain (NCRP) et le Comité Scientifique des Nations Unies (UNSCEAR, 1988). Commençons par examiner les raisons invoquées dans les deux cas.

TABLEAU I

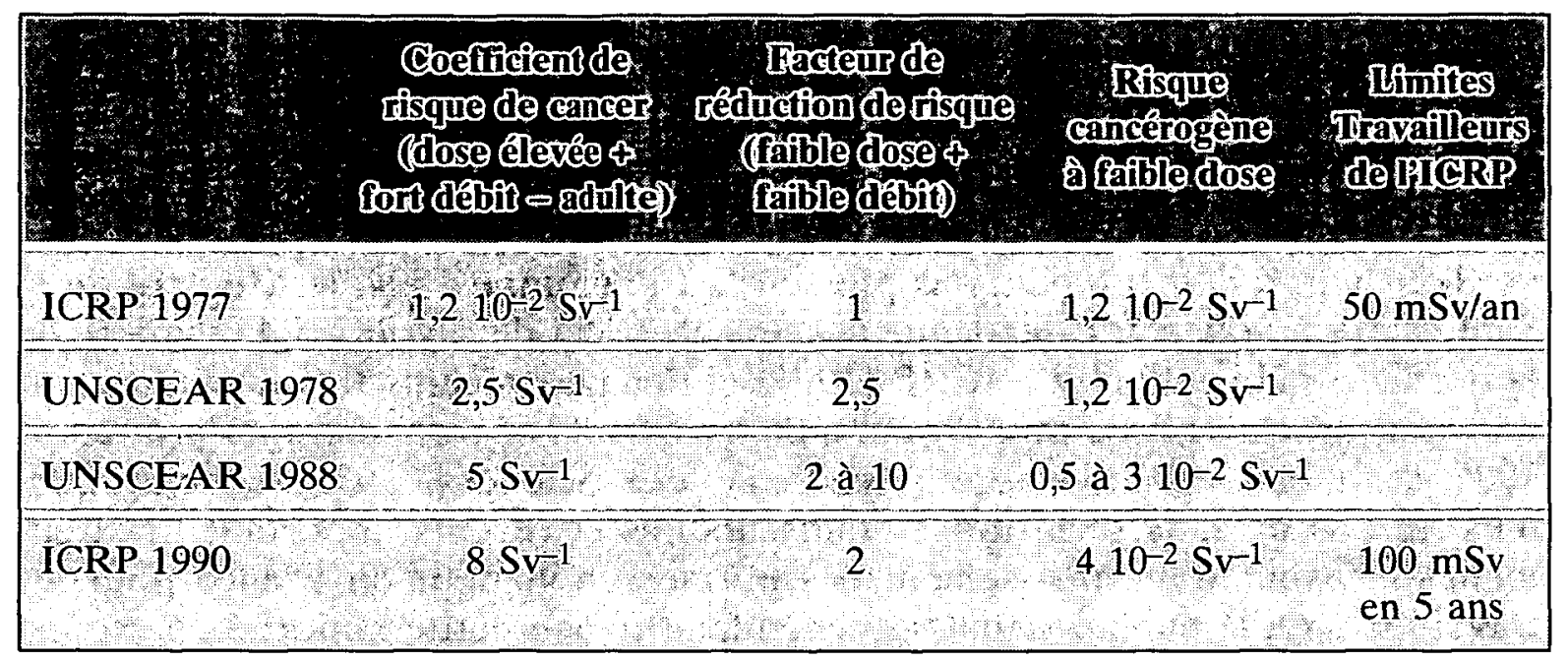

\subsection{Risque à fortes doses $(\geq 500 \mathrm{mSv})$}

\subsubsection{Un excès de cancers supérieur à celui attendu a été observé chez les survivants d'Hiroshima et Nagasaki $(\mathbf{H}+\mathbf{N})$}

Il est exact que 45 ans après l'explosion des bombes la fréquence des cancers solides en excès subsiste quoique faible : sur les 250000 survivants d'Hiroshima et Nagasaki $(\mathrm{H}+\mathrm{N})$ le nombre des cancers en excès par rapport à la population japonaise est inférieur à 1000 , mais la fréquence observée reste significativement supérieure chez des populations japonaises non irradiées (Shimizu et al., 1987; Thomson et al., 1994). De plus l'irradiation in utero et dans la prime enfance a entraîné un excès de la fréquence des cancers chez les sujets devenus adultes. Cependant l'augmentation du coefficient de risque cancérogène qui pourrait résulter de ces faits est relativement modeste, de l'ordre de 20 à $30 \%$ (Académie des Sciences, 1988).

Rappelons que la prééminence accordée aux survivants d' $\mathrm{H}+\mathrm{N}$ dans l'estimation de l'effet cancérogène introduit une surestimation de ce risque (Latarjet et Tubiana, 1989) sur lequel nous reviendrons. 


\subsubsection{La révision des doses reçues par les survivants des bombes atomiques}

L'estimation du risque cancérogène était avant 1986 fondée sur la dosimétrie effectuée en 1965 soit T65D. Ces doses ont été réévaluées en 1986 dans une nouvelle dosimétrie dite DS86. D'après celle-ci les doses gamma auraient été plus faibles que celles évaluées dans la T65D et la dose neutron, même à Hiroshima, pratiquement négligeable. Cette diminution des doses entraîne ipso facto une augmentation du risque cancérogène. Or si de nouvelles études confirment la dose gamma estimée en 1986, il n'en est pas de même pour la dose neutron qui semble avoir été notablement sous-estimée (Straume et al., 1992) ; la dose réelle se situant vraisemblablement entre les estimations T65 et DS86. Ce facteur d'augmentation du risque cancérogène est donc remis en question, d'autant qu'il subsiste des incohérences entre les doses reçues d'après DS86 et les réactions biologiques telles que les anomalies chromosomiques ou l'épilation post-irradiation et que la corrélation entre la survenue de leucémies et la chute des cheveux après l'explosion conforte la valeur de ce symptôme et accentue le sentiment d'une fiabilité insuffisante des estimations dosimétriques (Nerüshi et al., 1991 ; Stram, 1989).

\subsubsection{Le choix d'un modèle multiplicatif à coefficient constant pour estimer le risque sur toute la durée de la vie}

Environ la moitié des personnes irradiées à $\mathrm{H}+\mathrm{N}$ sont encore vivantes. Pour estimer le nombre global de cancers induits par l'irradiation on doit donc utiliser des modèles mathématiques extrapolant vers l'avenir. Mais si l'obligation d'utiliser un modèle de projection est indiscutable, beaucoup d'incertitudes subsistent quant au meilleur type de modèle (Fig. 1). Remarquons que l'introduction depuis 1986 des modèles de projection pour l'estimation des risques constitue la principale cause de l'augmentation du coefficient de risque à dose élevée puisqu'elle l'a multiplié par 2 ou par 3 (Académie des Sciences, 1988). Deux types de modèles de projection ont été proposés, les deux postulent qu'après une période de latence pendant laquelle la fréquence des cancers n'est pas augmentée, un excès de la fréquence des cancers persiste jusqu'à la mort. Dans le modèle dit de "risque absolu" (modèle additif) l'excès des cancers (appelons-le $A$ ) reste constant jusqu'à la fin de la vie. Après la période de latence la fréquence des cancers dans la population exposée est donc égale à la fréquence des cancers dans la population témoin $\mathrm{T}+A$ (Académie des Sciences, 1989).

Dans le modèle du "risque relatif" (modèle multiplicatif) ce qui est constant est non le nombre mais la proportion de cancers en excès. Si l'on appelle $R R$ ce facteur de risque relatif la fréquence de cancers dans la population exposée est égale à la fréquence des cancers de la population témoin $A$ multipliée par ce facteur de risque $R R$. Comme la fréquence des cancers croit rapidement avec l'âge, à partir de 40 ans, le vieillissement de la population entraîne donc un accroissement du nombre des cancers et le modèle multiplicatif prévoit donc une augmentation régulière du nombre de cancers en excès avec le temps (Fig. 1). 


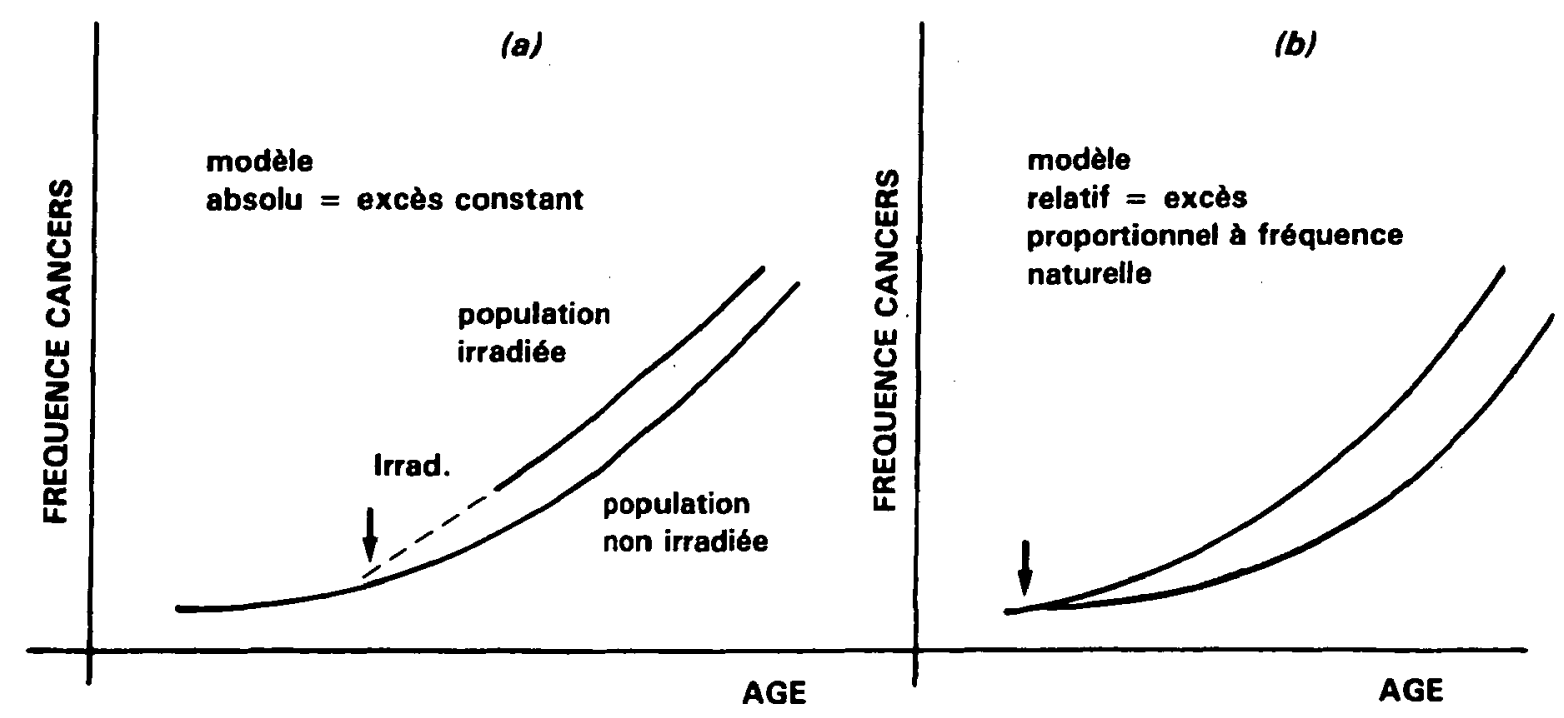

(c)

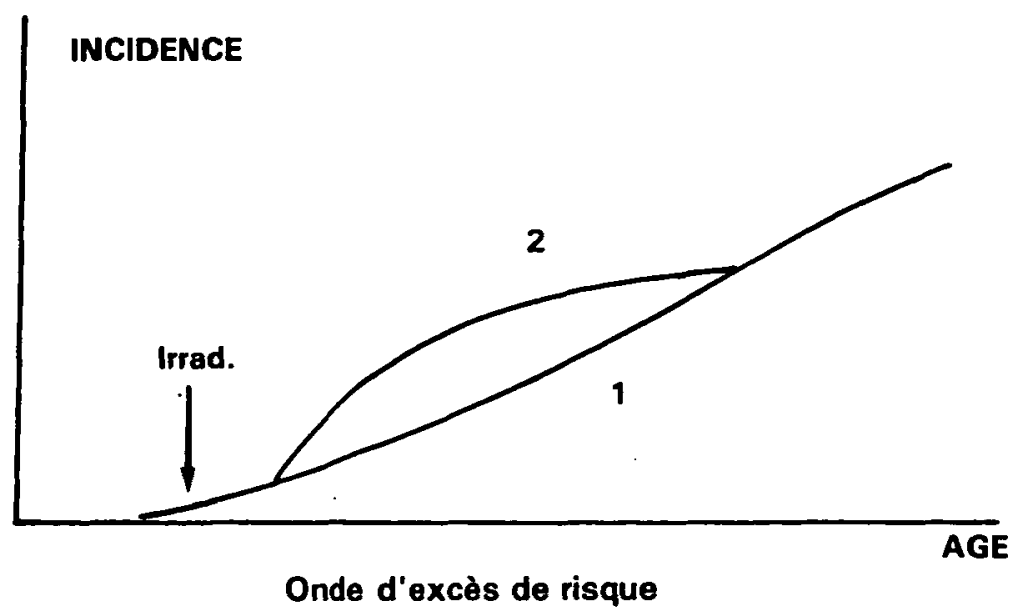

Fig. I - Modèle de projection du risque cancérogène. La fréquence naturelle des cancers très basse au dessous de 40 ans augmente ensuite rapidement avec l'âge. Dans le modèle additif à risque constant après l'irradiation il s'ajoute aux cancers spontanés un nombre supplémentaire constant de cancers jusqu'au décès. Dans le modèle multiplicatif la fréquence est multipliée par un facteur constant. De ce fait le nombre de cancers en excès augmente rapidement avec l'âge en même temps que la fréquence naturelle crô̂t. Dans un troisième modèle qui est celui dont la validité a été prouvée pour les leucémies et les ostéosarcomes, il s'ajoute à la fréquence de base une onde d'excès de cancers pendant une quinzaine d'années. Il est possible que ce dernier modèle soit aussi valable pour d'autres cancers. 1) population non irradiée; 2) population irradiée.

The natural incidence of cancer is very low under the age of 40, but then increases rapidly with age. After irradiation, in the additive risk model a constant absolute number of cancers until death is added to spontaneous cancers. In the multiplicative model, the incidence is multiplied by a constant factor. For this reason; the excess number of cancers increases rapidly with age at the same time as the increase in natural incidence. In a third model, which proved valid in leukemias and osteosarcoma, an excess of cancers during fifteen years is added to the basic incidence. It is possible that this last model may be valid for other cancers. I) non-irradiated population; 2) irradiated population. 
L'ICRP a choisi en 1990 (ICRP, 1991) le modèle du risque relatif à coefficient constant parce qu'il lui paraissait mieux correspondre à plusieurs données mais aussi parce qu'il était le plus prudent. Remarquons que non seulement pour les leucémies mais aussi pour d'autres types de tumeurs, comme les ostéosarcomes (BEIR IV, 1988), le risque après être passé par un maximum, revient après une dizaine d'années à une valeur proche de la normale (Fig. 1). Il en est de même dans certaines études effectuées sur des malades irradiés où la fréquence des cancers est redevenue normale environ 25 ans après l'irradiation (Darby et al., 1987) (Fig. 2). Pour les autres types de cancers c'est tantôt le modèle absolu, tantôt le relatif qui donne le moins mauvais ajustement ; l'utilisation d'un seul modèle pour tous les cancers est une simplification abusive.

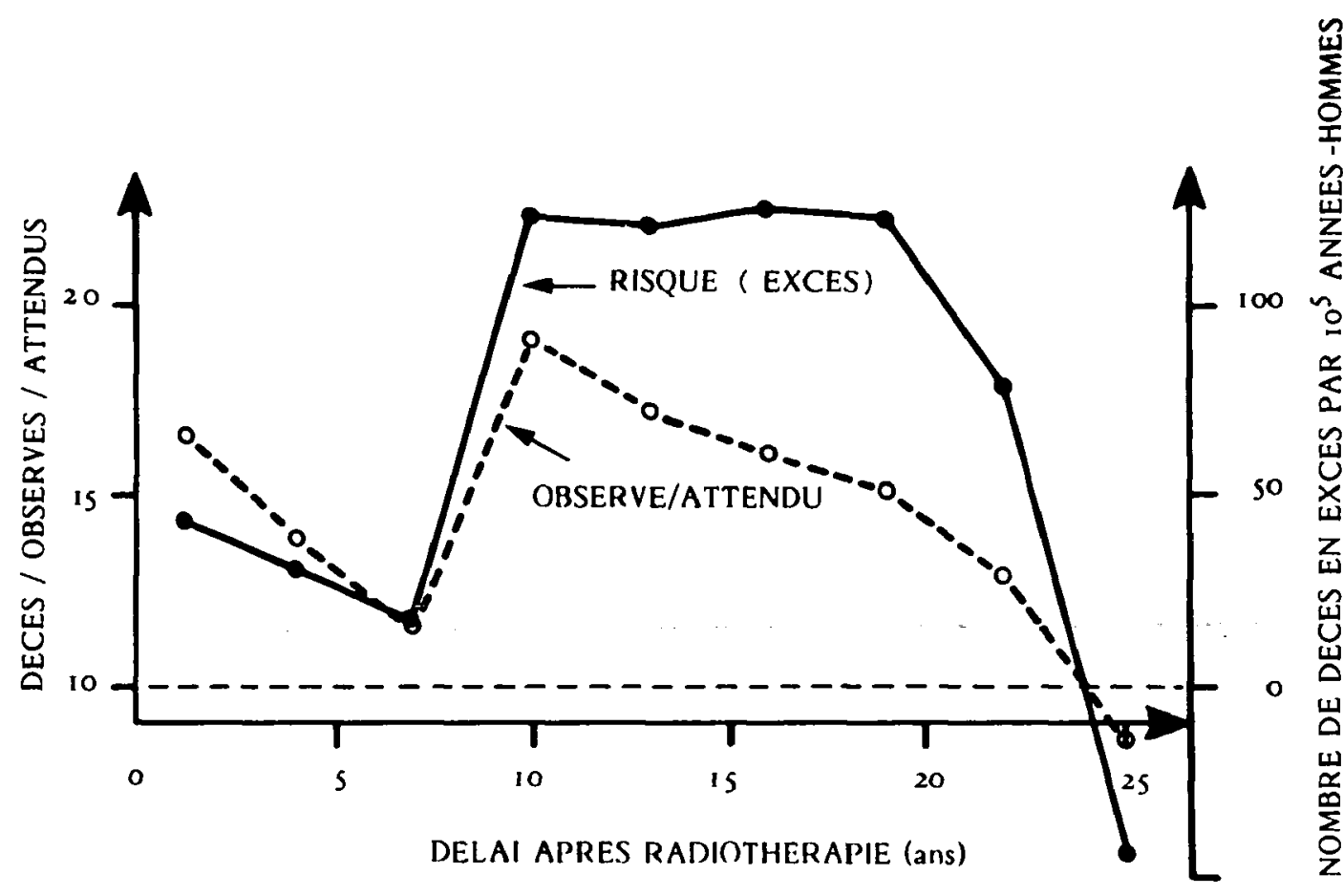

Fig. 2 - Fréquence des cancers excédentaires en fonction du délai après irradiation, chez des malades traités pour spondylarthrite ankylosante. On a calculé, d'une part le rapport fréquence observéelfréquence attendue, et d'autre part le nombre de cancers en excès (nombre observé moins nombre attendu rapporté au nombre de personnes exposées au risque dans la population). Le nombre de leucémies a été maximum 3 à 5 ans après irradiation puis a diminué. Le nombre de tumeurs solides dans les régions irradiées a été maximal 9 à 11 ans après irradiation, il a diminué après 20 ans, mais cette diminution se fait à des rythmes différents pour les divers types du cancer.

Excess deaths due to malignant disease as a function of time after irradiation in patiens treated for ankylosing spondylitis (number observed minus number expected related to number of persons exposed to risk in the population). The number of leukemias reached a maximum 3 to 5 years after irradiation, and then diminished. The number of solid tumors in the irradiated region was at a maximum 9 to 11 years after irradiation and diminished after 20 years. (After Darby, Doll et al., 1987). 
De plus certaines données sont en contradiction avec l'hypothèse d'une modèle multiplicatif (Chau, 1987). Ainsi le nombre de cancers du sein en excès (à dose égale) est voisin chez les Japonaises irradiées à $\mathrm{H}+\mathrm{N}$ et les Canadiennes ou Américaines irradiées pendant les radioscopies effectuées pour surveiller un pneumothorax thérapeutique, alors que la fréquence des cancers du sein est environ cinq fois plus grande en Amérique du Nord qu'au Japon (Schneider, 1995). Cette observation s'accorde bien avec un modèle absolu mais est difficilement compatible avec un modèle de risque relatif.

Enfin depuis 1991 il a été montré que le modèle de risque relatif à coefficient constant était en contradiction avec les observations effectuées sur les survivants de $\mathrm{H}+\mathrm{N}$ notamment chez les sujets irradiés pendant l'enfance chez qui une diminution significative est observée (Little et al., 1991); Celle-ci est retrouvée chez des enfants irradiés pour des raisons médicales (De Vathaine et al., 1995) et existe également quoique moins importante chez les sujets irradiés à l'âge adulte soit à $\mathrm{H}+\mathrm{N}$, soit pour des raisons médicales (Curtis et al., 1994) (Fig. 3). La validité du modèle choisi par l'ICRP est donc remise en cause, or ce modèle est, de très loin, le principal facteur ayant provoqué la multiplication par 3 du risque cancérogène (Académie des Sciences, 1988). Chez les mineurs exposés professionnellement au radon, le risque de cancers du poumon décroît aussi avec l'âge, ce qui est incompatible avec le modèle à coefficient constant (UNSCEAR, 1994).

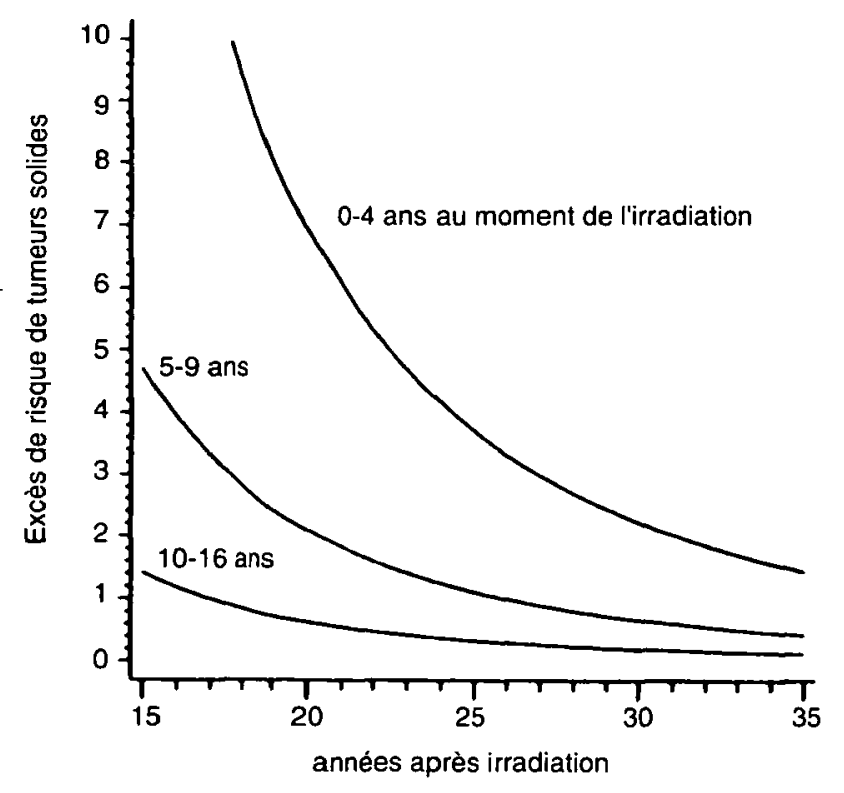

Fig. 3 - Excès de risque relatif d'apparition de cancers solides chez les enfants irradiés $\grave{a}$ des âges entre 0 et 16 ans. Pour ces groupes d'enfants on observe une diminution statistiquement significative du risque après un délai supérieur à 15 ans, cependant l'excès ne devient pas nul même 35 ans après irradiation (d'après de Vathaire et al., 1995.) Une même diminution du risque a été observée chez les adultes.

Excess relative risk of occurrence of solid tumors in children irradiated between the ages of 0 and 16. For these groups of children a statistically significant reduction in risk is observed after a delay longer. than 15 years. However, the excess does not become nil even 35 years after irradiation (de Vathaire et al.). A relative reduction in risk was also observed in adults. 
Le choix du modèle de projection est d'autant plus crucial que dans le modèle multiplicatif, les dernières années de vie sont les plus pénalisantes. Avec le modèle de l'ICRP (1991) la moitié des cancers prévus surviendraient après 75 ans (donc à un âge supérieur à l'espérance de vie des hommes européens) (Fig. 4).

Les justifications de l'accroissement du risque cancérogène à forte dose sont donc fragiles, elles le sont d'autant plus que l'écart entre les estimations fondées soit sur les survivants de $\mathrm{H}+\mathrm{N}$, soit sur d'autres enquêtes notamment les malades irradiés, loin de s'atténuer se sont accentuées depuis cinq ans.

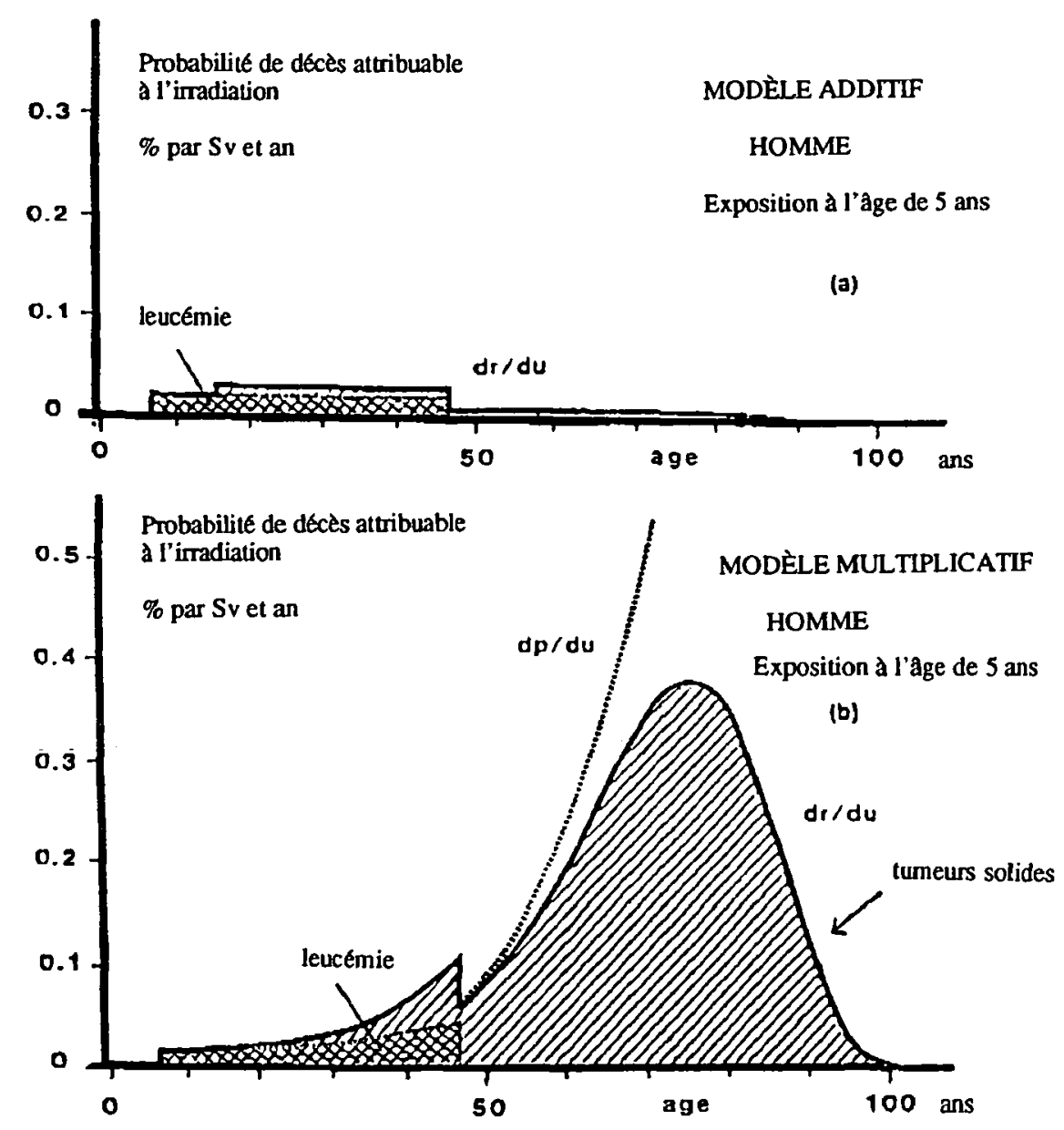

Fig. 4 - Répartition en fonction de l'âge des taux de décès par leucémies et cancers en excès calculés avec soit un modèle additif (risque absolu courbe du haut) soit multiplicatif à coefficient constant (risque relatif courbe du bas). Ces courbes théoriques, extraites du rapport 60 de l'ICRP, concernent un groupe d'enfants irradiés à l'âge de 5 ans. Comme on le voit avec le modèle multiplicatif le nombre de cancers en excès est plus grand et la moitié des cancers en excès apparaît à un âge supérieur à celui de l'espérance de vie des français (73 ans).

Distribution in function of age of mortality rates by excess leukemias and cancers calculated with the additive model (absolute risk- top curve) and the multiplicative model with constant coefficient (relative risk-bottom curve). These theoretical curves, taken from the ICRP Report 60, concern a group of children irradiated at the age of 5. As can be seen with the multiplicative model, the number of excess cancers is larger and half of the excess cancers occur at an age older than the mean expected life span. 
Comme on le voit sur le tableau II (UNSCEAR, 1988) dans plusieurs enquêtes les cancers observés sont notablement moins nombreux que ceux prédits par le coefficient de risque utilisé, qui était lui-même à l'époque notablement inférieur à celui proposé aujourd'hui. Pour les leucémies leur fréquence était nettement inférieure à celle calculée chez les 85000 femmes traitées pour cancer du col utérin en utilisant le précédent facteur de risque leucémogène (Boice et al., 1987). Dans la série des 110000 femmes traitées pour cancer du corps utérin (Curtis et al., 1994), la différence entre le nombre de leucémies prédit et celui observé est hautement significative ; chez les malades irradiés par curiethérapie, à faible débit de dose $(0,4 \mathrm{mSv} /$ minute), le coefficient de risque cancérogène est environ 4 fois plus faible que celui obtenu par la cohorte Hiroshima et Nagasaki ce qui confirme le rôle important du débit de dose.

TABLEAU II*

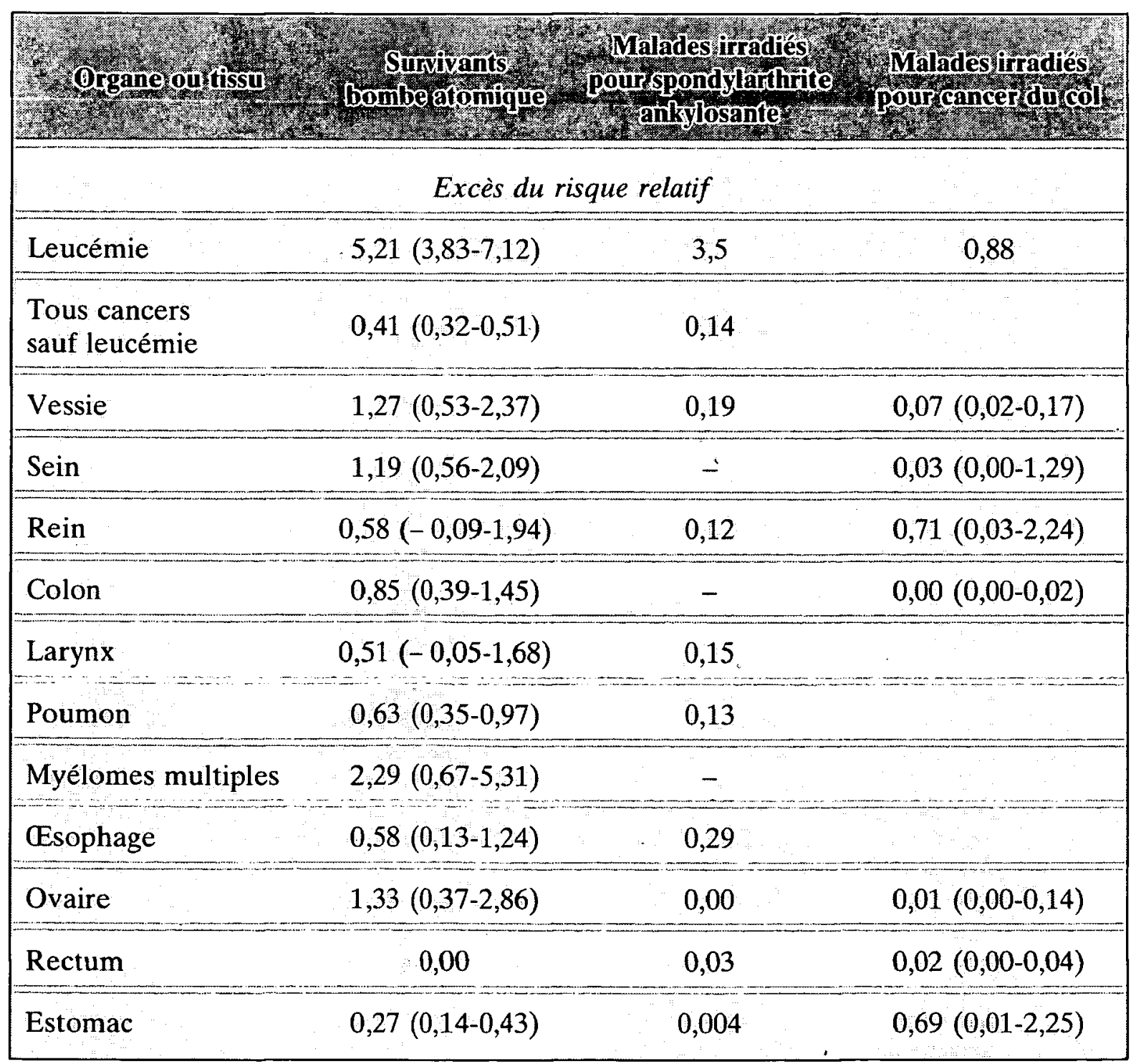

* D'après UNSCEAR (1988). 
Les figures 5-8 extraites du rapport de l'UNSCEAR (1994) montrent que pour presque toutes les localisations l'effet cancérogène est à dose égale plus grand chez les sujets irradiés à $\mathrm{H}+\mathrm{N}$ que dans les autres groupes. L'explication la plus plausible pour ces différences est le débit de dose, qui a été beaucoup plus élevé à $\mathrm{H}+\mathrm{N}$ que chez les malades irradiés.
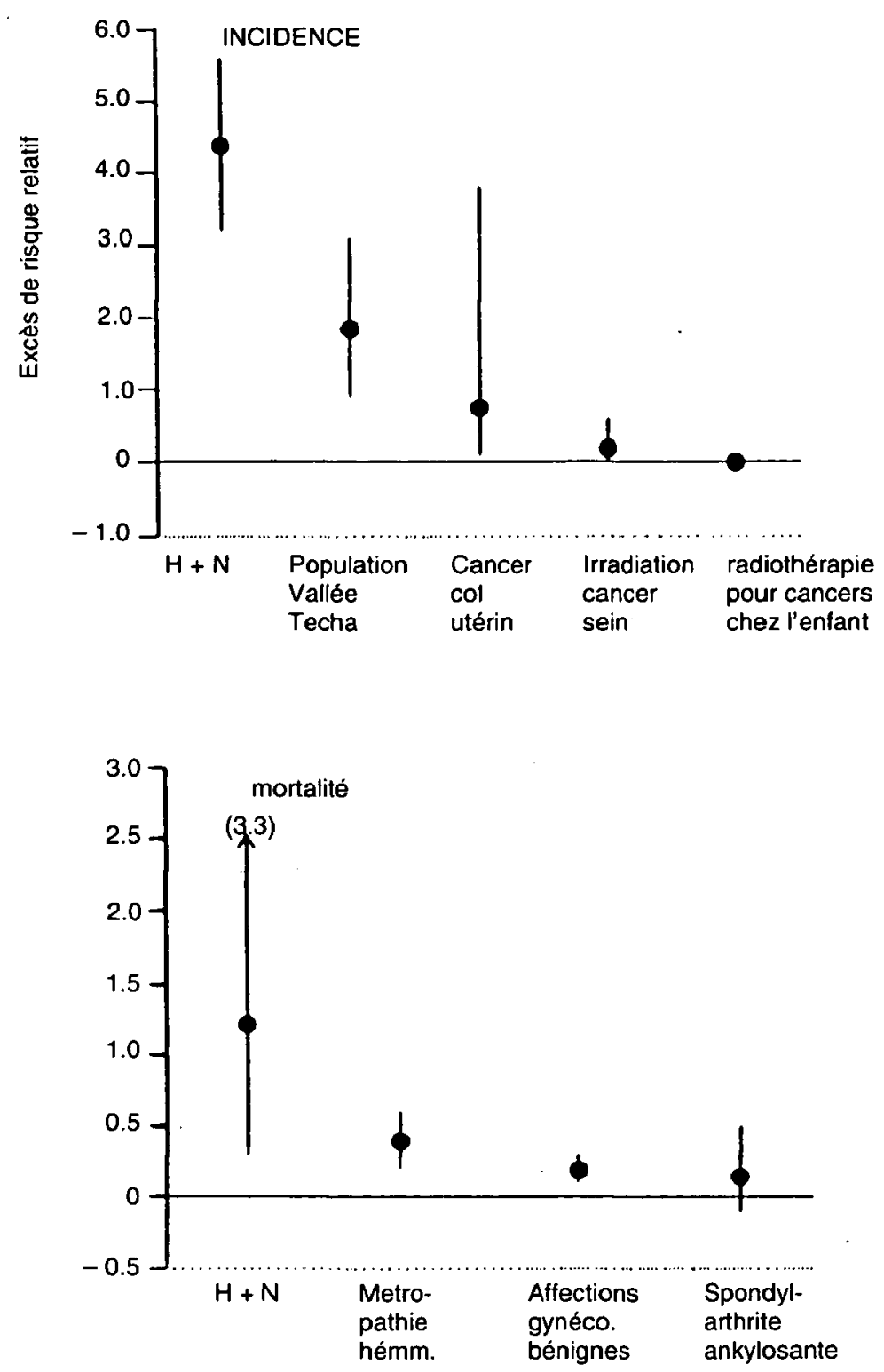

Fig. 5 - Comparaison de l'effet leucémogène dans la cohorte des sujets irradiés à Hiroshima et Nagasaki $(\mathrm{H}+\mathrm{N})$ et dans divers autres groupes de sujets irradiés. L'effet leucémogène à dose égale (par Sv) est nettement plus élevé chez les irradiés à $\mathrm{H}+\mathrm{N}$, sans doute en raison du débit de dose plus élevé (d'après rapport UNSCEAR, 1994).

Comparison of the leukemogenic effect in a cohort of subjects irradiated in Hiroshima and Nagasaki $(\mathbf{H}+\mathbf{N})$ and in various other groups of irradiated subjects. The leukemogenic effect at equal dose (by Sv) is markedly higher in subjects irradiated in $\mathrm{H}+\mathrm{N}$, most probably because of the higher dose-rate (after the UNSCEAR 1994 report). 

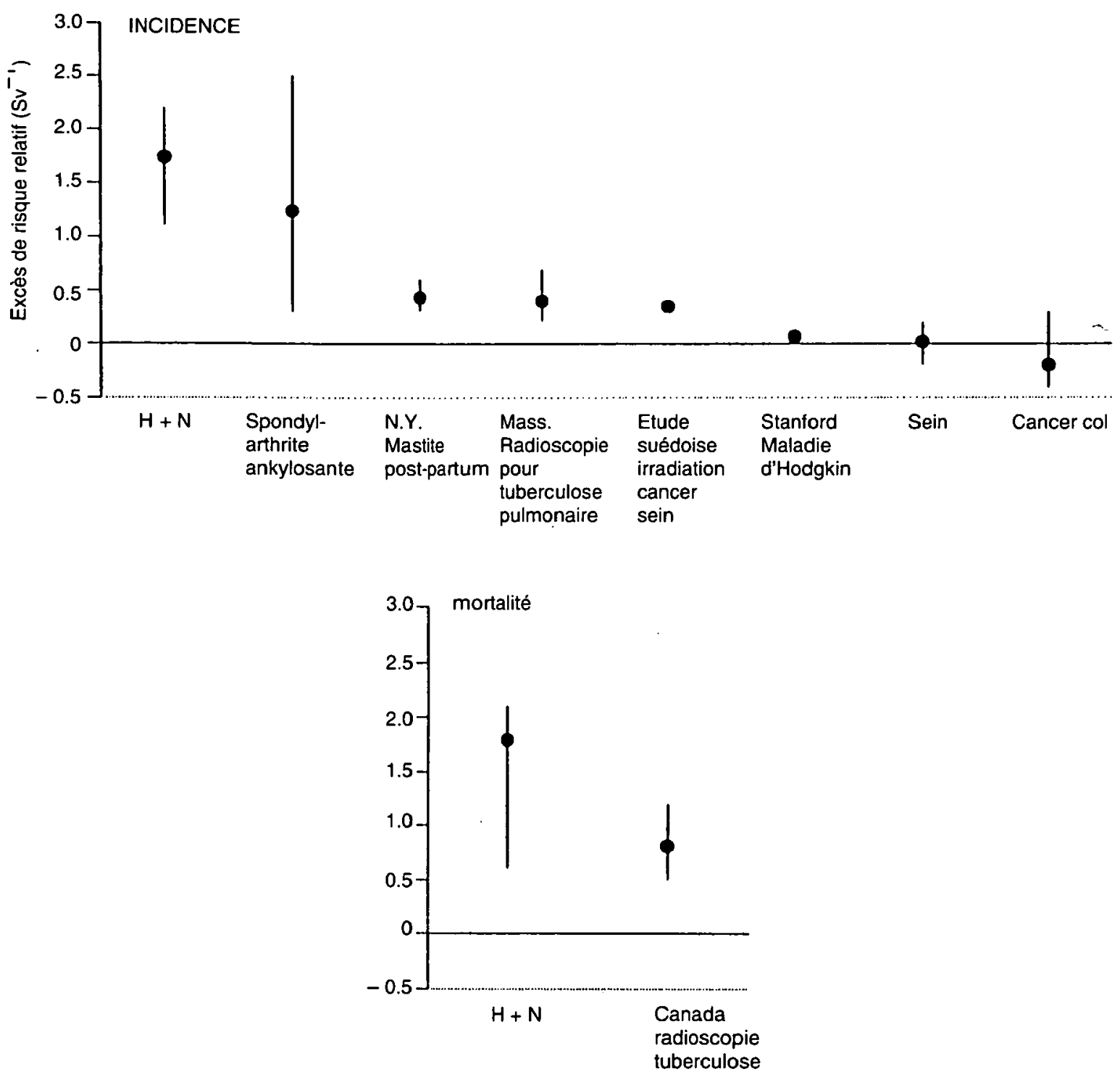

Fig. 6 - Comparaison de l'excès de risque relatif pour les cancers du sein chez divers groupes de sujets irradiés notamment la cohorte $\mathrm{H}+\mathrm{N}$. A l'origine de ces écarts on invoque le débit de dose très élevé lors des explosions atomiques (d'après rapport UNSCEAR, 1994).

Comparison of the relative excess risk for cancers of the breast in various groups of irradiated subjects, notably the cohort $\mathrm{H}+\mathrm{N}$. The high dose rate during the atomic explosions is probably the reason for these variations. 

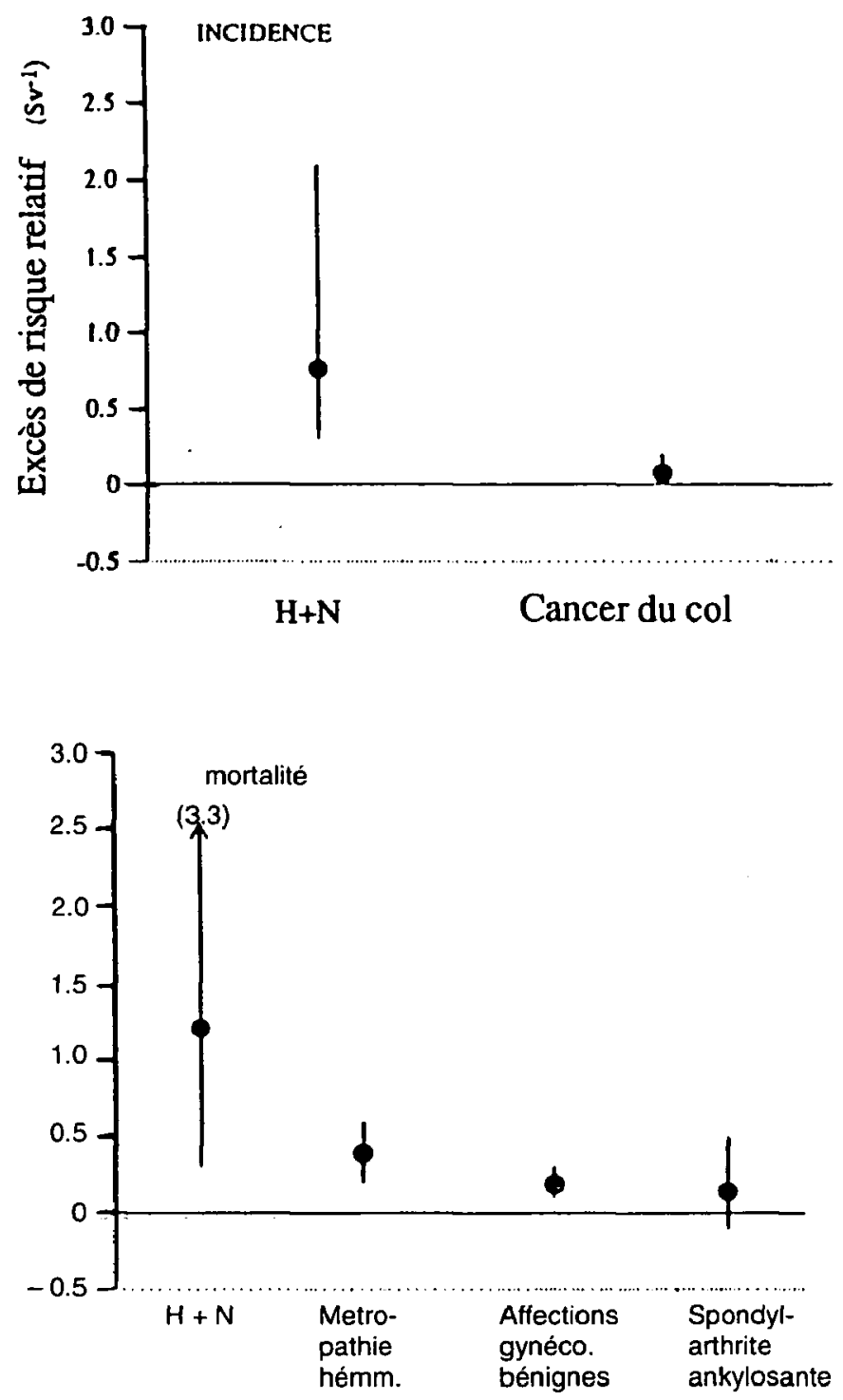

Fig. 7 - Comparaison de l'excès de risque relatif pour les cancers de la vessie chez divers groupes de sujets irradiés notamment la cohorte $\mathbf{H}+\mathbf{N}$. A l'origine de ces écarts on invoque le débit de dose très élevé lors des explosions atomiques (d'après rapport UNSCEAR, 1994).

Comparison of the relative excess risk for cancers of the bladder in various groups of irradiated subjects, notably the cohort $\mathrm{H}+\mathrm{N}$. The high dose rate during the atomic explosions is probably the reason for these variations. 


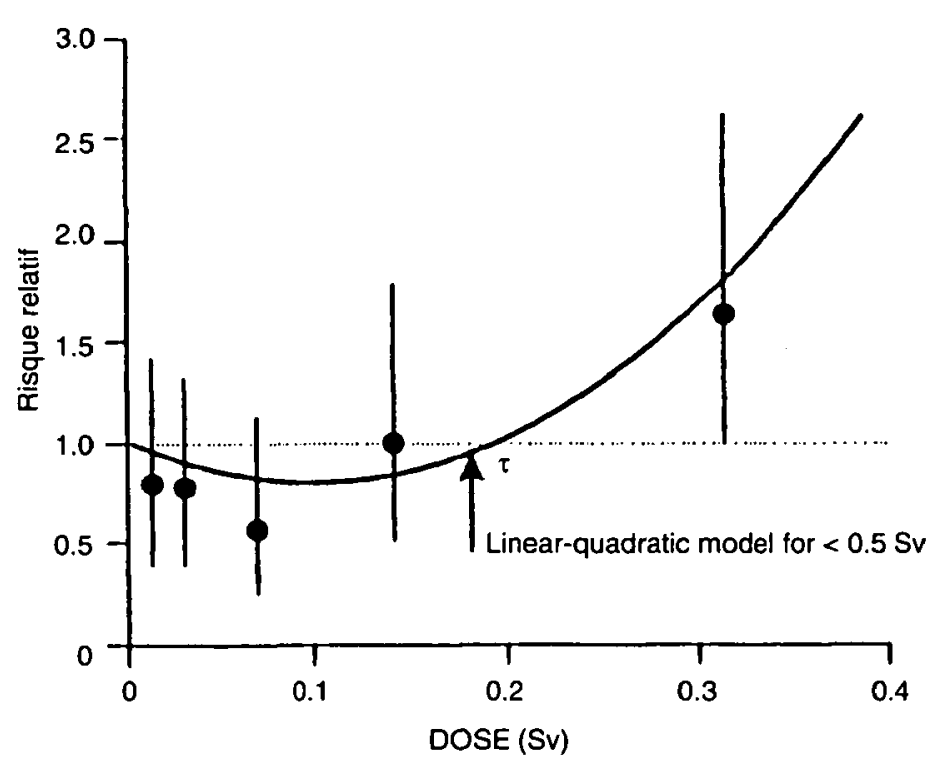

Fig. 8 - Relation entre la dose et la fréquence de leucémie chez les sujets irradiés à Hiroshima et Nagasaki (d'après Shimuzu et al., 1992 et UNSCEAR, 1994). Aucune augmentation n'est constatée pour les doses inférieures à $200 \mathrm{mSv}$; on observe même une légère diminution de la fréquence pour des doses inférieures à $100 \mathrm{mSv}$ mais celle-ci n'est pas statistiquement significative.

Relation between the dose and incidence of leukemia in subjects irradiated in Hiroshima and Nagasaki (from Shimuzu et al., 1992; UNSCEAR, 1994). No increase is observed for doses lower than $200 \mathrm{mSv}$; even a slight decrease in the incidence is observed for doses lower than $100 \mathrm{mSv}$, but this is not statistically significant.

\subsection{Extrapolation sur les faibles doses (< $200 \mathrm{mSv})$}

Considérons maintenant le deuxième facteur ayant entraîné l'augmentation du risque à faible dose : le choix de la valeur 2 pour le coefficient de réduction du risque à faible dose, faible débit.

En 1980 un rapport du NCRP, en 1988 un autre de l'UNSCEAR avaient conclu, au terme d'une analyse circonstanciée des données expérimentales et humaines disponibles, que la valeur de ce coefficient variait significativement selon le phénomène étudié et se situait dans une fourchette allant de 2 à 10 . Il était de plus reconnu que pour certains effets cancérogènes à faible dose-faible débit, ce coefficient pouvait être supérieur à 10 . Pour avoir participé aux travaux de ces deux groupes, je peux témoigner du sérieux des analyses qui ont nécessité des dizaines d'heures de réunion. En 1989 l'ICRP (1991) a choisi la valeur 2, la plus péjorative. La justification de ce choix est un souci de prudence. En effet la plupart des données expérimentales concernant les irradiations délivrées avec un très faible débit sont proches de 4 ou 5 (Tubiana et al., 1992). Ainsi dans l'étude de Thomson et Grahn (1989) comparant une exposition unique à une exposition chronique à très faible débit de dose, la valeur est 
de 5. Dans l'étude de Morin et al. (1990) le coefficient est également de 5 pour la plupart des cancers quand le débit de dose passe de $80 \mathrm{mSv} / \mathrm{h}$ à $1,3 \mathrm{mSv} / \mathrm{h}$. De plus il est surprenant de trouver, parmi les données retenues pour justifier un coefficient de réduction égal à 2 , des valeurs non mesurées expérimentalement mais calculées en utilisant la méthode proposée par Pierce et Vaeth (1989). Celle-ci postule que la relation entre la dose et l'effet cancérogène est linéaire-quadratique, donc on calcule la pente initiale de la courbe, dans la zone des doses pour lesquelles aucun effet n'est observé, en recherchant quelle est la valeur des paramètres de la fonction linéaire quadratique qui ajuste les données épidémiologiques obtenues pour des doses supérieures à $0,5 \mathrm{~Sv}$. Cette méthode de calcul n'aurait de sens que si la validité du modèle linéaire-quadratique avait été prouvée ; comme il n'en est rien elle constitue une véritable tautologie.

De façon plus générale depuis 20 ans la discussion sur les effets à faible dose-faible débit est rendue confuse par l'utilisation du modèle linéaire-quadratique. Celui-ci mérite donc discussion d'autant que très rares sont les données expérimentales qui peuvent être ajustées avec lui. Ce modèle avait été proposé, il y a une vingtaine d'années, à partir de considérations microdosimétriques. L'énergie des électrons mis en mouvement est délivrée au milieu irradié par paquets de dimensions variables (entre 15 et $100 \mathrm{eV}$ ) (Goodhead et al., 1993; Goodhead, 1994 ; Tubiana et al., 1990). Comme la molécule cible est l'ADN on a pensé que comme la rupture d'un seul brin est rapidement réparée l'effet biologique était déterminé principalement par la rupture des deux brins de la molécule. Celle-ci peut-être causée soit par la rupture successive des deux brins survenant à courte distance et dans un court intervalle de temps (sommation, pour couper les deux brins de la molécule d'ADN, des effets indépendants de deux petits paquets d'énergie), soit par la rupture non réparable des deux brins causée par un gros paquet d'énergie. Ce modèle rendait bien compte des mutations induites par l'irradiation d'une plante, la Tradescantia, pour laquelle il était possible de mesurer l'effet mutagène à partir de doses très faibles, de $1 \mathrm{mSv}$, jusqu'à des doses très élevées (NCRP, 1980). Il parut être conforté par la relation dose-effet pour l'induction de chromosomes dicentriques et les courbes de survie des cellules irradiées. Le modèle parut donc satisfaisant, il eut un immense succès bien que :

1) aucun argument expérimental ou épidémiologique n'ait démontré sa validité pour l'induction de cancers; au contraire la quasi totalité des données expérimentales ne peuvent pas être ajustées avec ce modèle,

2) d'autres modèles peuvent rendre compte aussi bien ou mieux de la courbe de survie des cellules (Tubiana et al., 1990),

3) pour la plupart des aberrations chromosomiques, les relations dose-effet ne s'ajustent pas avec ce modèle (Le François et al., 1989).

Pour tester la validité d'une théorie ou d'un modèle, l'usage en science est de rechercher si ses prédictions sont en accord avec les faits. Les plus importantes prédictions du modèle sont que comme l'effet cancérogène dans le 
domaine des faibles doses est la conséquence d'un seul événement physique ayant déterminé une lésion irréversible : 1) toute dose, si faible soit-elle, comporte un risque cancérogène et la relation est, dans ce domaine de dose, linéaire, 2) dans ce domaine de dose l'effet n'est pas influencé par le débit.

La première prévision n'a jamais pu être prouvée, bien au contraire de nombreux arguments donnent à penser qu'il en est autrement (Académie des Sciences, 1995). Quant à la deuxième elle est contredite par les faits puisque même aux doses les plus faibles pour lesquelles un effet cancérogène ait été observé, cet effet est considérablement réduit aux faibles débits (Masse, 1990 ; Morin et al., 1990 ; Tubiana et al., 1990). Il en est de même pour les irradiations effectuées avec des particules alpha (Morlier et al., 1992) bien que dans ce cas le transfert d'énergie se fasse par paquets importants (Goodhead et al., 1993 ; Goodhead, 1994 ; Tubiana et al., 1990) qui sont ceux déterminant des lésions ayant la plus grande probabilité d'être irréparables et donc pour lesquels, d'après le modèle linéaire quadratique, l'effet devrait être indépendant du débit.

Ces contradictions font qu'il n'est plus légitime d'admettre ce modèle et de fait il a été vigoureusement contesté par de nombreux auteurs (Abelson, 1994 ; Koshland, 1994 ; Pollycove, 1995 ; Thiessen, 1991; Tubiana et al., 1992; Walinder, 1995). Si l'on exclut, de ce fait, les pentes à l'origine calculées avec la méthode de Pierce et Vaeth (1989) le choix d'un facteur de réduction égal à 2 est encore davantage sujet à caution. De même, si l'on remet en question ce dogme, il n'est plus justifié d'utiliser un seul facteur de réduction pour à la fois la dose et le débit de dose. On peut enfin se demander s'il est raisonnable d'utiliser un seul facteur de réduction entre 0 et $500 \mathrm{mSv}$ et s'il ne serait pas opportun au minimum de distinguer les faibles doses $(<200 \mathrm{mSv})$ et les très faibles doses $(<20 \mathrm{mSv})$.

De plus le modèle prédit que l'on peut calculer le nombre de cancers induits à partir de la dose collective (en homme-Sievert) ce qui n'a jamais été observé et est très peu plausible. Enfin si la lésion d'une seule cellule était à l'origine du cancer, la gravité de celui-ci devrait être indépendante de la dose, or ce n'est pas ce que l'on observe, et qui suggère une plus grande gravité des cancers apparus après des doses élevées ou des irradiations répétées (Walinder, 1995) ; ceci peut s'expliquer aisément si l'on admet le rôle de la prolifération cellulaire.

Le problème de l'extrapolation des effets cancérogènes à fortes doses vers les faibles doses se pose donc à nouveau avec acuité (Figs. 9 et 10). Pour l'aborder le Rapport de l'Académie des Sciences (1995) discute successivement les données fondamentales puis les données épidémiologiques. Nous suivrons ce plan. 


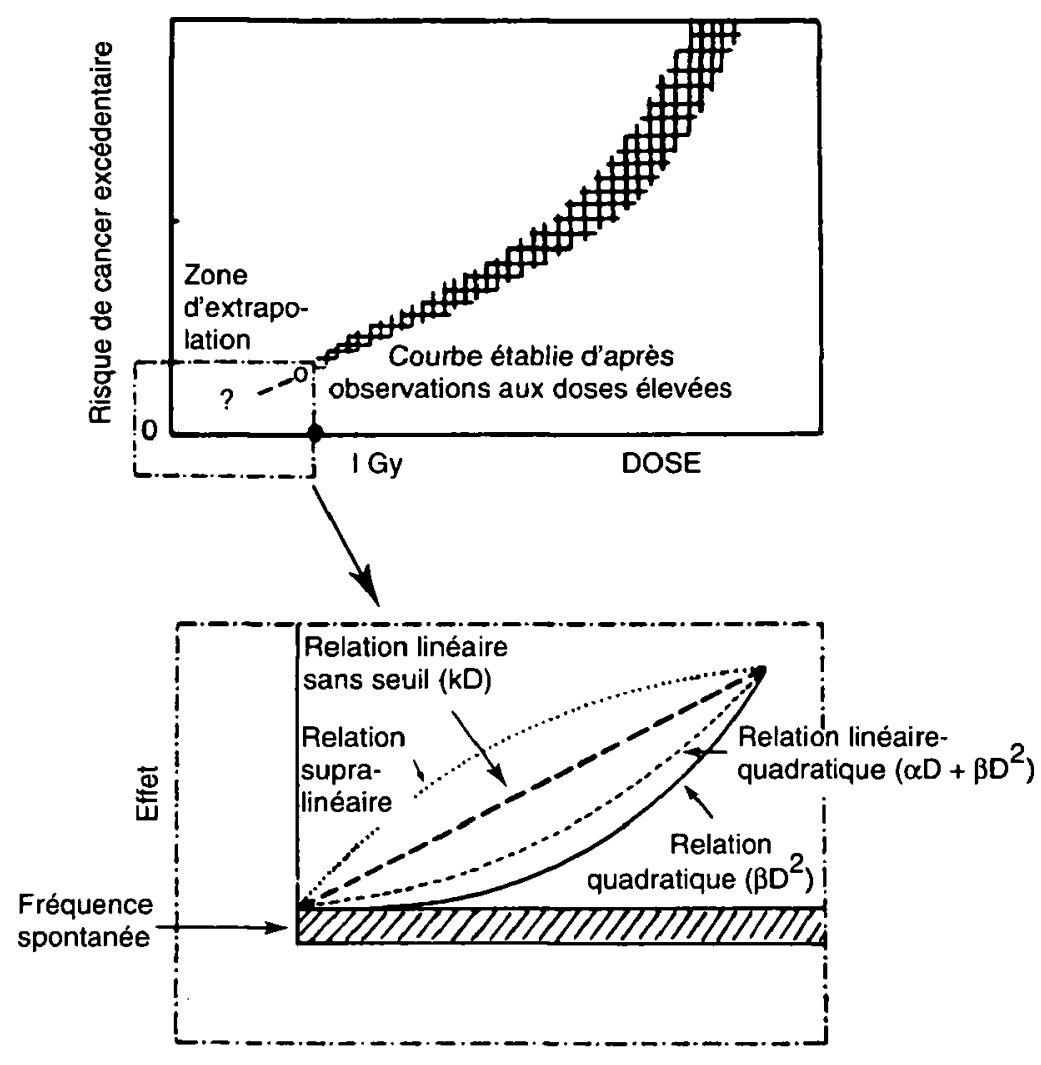

Fig. 9 - Extrapolation de la relation dose-effet vers les faibles doses. Quelques enquêtes ont permis d'établir la relation dose-effet pour les doses supérieures à $0,5 \mathrm{~Sv}$; aucune n'a permis d'estimer avec une fiabilité suffisante le risque pour des doses inférieures. Or, c'est l'effet de ces faibles doses qui est important du point de vue de la radioprotection. Pour parvenir à l'estimer, il faudrait connaître la forme de la relation dose-effet dans cette gamme de doses, de façon à pouvoir extrapoler entre la fréquence naturelle et la fréquence observée après une irradiation de $1 \mathrm{~Sv}$. La figure montre que l'évaluation du risque est différente selon la fonction choisie. Hormis l'hypothèse d'une relation supra-linéaire que les récentes données permettent d'exclure, la relation linéaire est celle qui prévoit le risque le plus élevé, environ double, pour les doses inférieures à $0,5 \mathrm{~Sv}$, de celui prévu par la relation linéaire quadratique. La relation quadratique qui semble scientifiquement plus plausible (en raison des observations faites après irradiation par particules alpha) donne des prévisions inférieures.

Extrapolation of the dose-effect relation to low doses. Several studies have established a dose-effect relation with doses greater than $0.5 \mathrm{~Sv}$, but none have provided accurate data below $0.5 \mathrm{~Sv}$. The effect of these low doses is the relevant factor in radiation protection. For this extrapolation till a dose zero it is necessary to know the shape of the dose-effect relation. The figure shows how much the risk evaluated depends on the function chosen. The linear relation gives the greatest risk which, at $0.5 \mathrm{~Sv}$, is about double of that given by the linear quadratic relation and much higher than a quadratic function. 


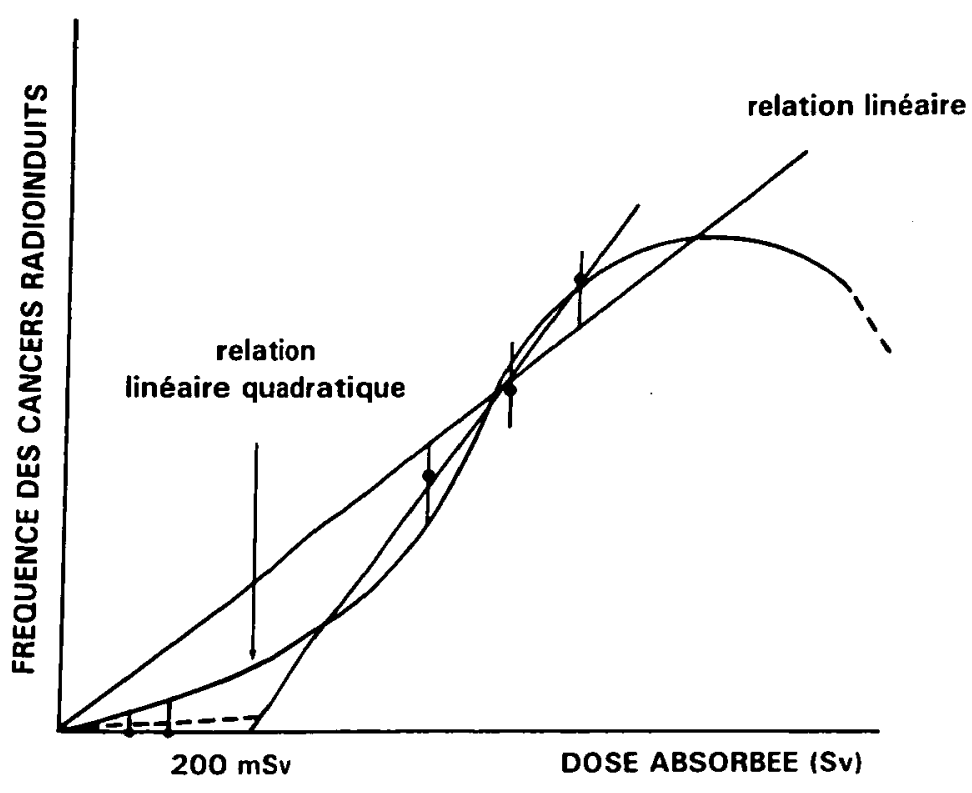

Fig. 10 - L'utilisation d'une relation linéaire quadratique pour estimer le risque dans le domaine des faibles doses aboutit à une estimation qui est contestable. En effet si l'on extrapole une droite passant par des points correspondant aux données épidémiologiques on aboutit fréquemment à un seuil correspondant à une dose d'environ $200 \mathrm{mSv}$, dose au dessous de laquelle d'ailleurs aucune étude épidémiologique n'a pu mettre en évidence un effet cancérogène. L'effet réel (courbe en pointillé) pourrait être très inférieur, s'il existait une discontinuité dans l'efficacité de l'effet cancérogène au dessous de quelques centaines de mSv, notamment en raison de la réparation des lésions de l'ADN.

The use of the linear quadratic relation to estimate risk for low doses leads to a doubtful estimation. A linear extrapolation based only on the values given by the epidemiologic data at high doses crosses generally the horizontal axis at a dose of approximately $200 \mathrm{mSv}$, a dose under which no epidemiological study has shown evidence of a carcinogenic effect. The actual effect (dotted curve) could be much lower than a linear quadratic curve starting at zero if there is a discontinuity in the efficacy of the carcinogenic effect below $200 \mathrm{mSv}$, notably due to DNA repair.

\section{Données fondamentales récentes}

\subsection{Lésions de l'ADN et système de réparation}

L'effet biologique des rayonnement ionisants est pour l'essentiel, notamment la mortalité cellulaire et l'effet cancérogène, dû à des lésions irréversibles des molécules d'ADN. Ces lésions sont causées d'une part par des agents d'oxydation formés lors de l'interaction entre les rayonnements et l'eau des tissus (peroxydes, radicaux hydroxyles, eau oxygénée...) qui déterminent divers types de lésions, notamment des cassures d'un seul brin et plus rarement des deux brins de l'ADN ainsi que des pontages. D'autre part les transferts d'énergie directs à l'intérieur des molécules d'ADN ou des chromosomes provoquent également des lésions voisines de celles causées par effet indirect et dont la gravité est fonction de l'importance du paquet d'énergie mis en jeu (Tubiana et al., 1990). On considère que $1000 \mathrm{mSv}$ délivrés en un temps court 
causent 1000 cassures simple brin et 40 double-brin (Académie des Sciences, 1995). Or du fait du métabolisme normal d'une cellule il s'y forme des agents d'oxydation qui déterminent aussi un grand nombre de lésions de l'ADN : chaque seconde environ 2 cassures simple-brin et un pontage. Le nombre de cassures double-brins est beaucoup plus petite : environ 1 cassure double brin pour 15000 cassures simple-brin. D'après ces estimations il surviendrait normalement dans une cellule une dizaine de cassures double-brin chaque jour. Ces lésions seraient incompatibles avec la vie si elles n'étaient pas réparées; mais il existe dans les cellules de puissants systèmes de réparation de l'ADN qui ont fait l'objet depuis 20 ans de nombreux travaux, (Cleaver, 1994; Koshland et al., 1994 ; Koshland, 1994 ; Tubiana et al., 1990) notamment parce que des défauts de ces systèmes sont à l'origine d'un grand nombre de maladies (xeroderma, ataxia teleangectasia, etc.) caractérisées 1) par une hypersensibilité aux rayons ultra-violet ou aux rayons $X$ et 2 ) associées pour la plupart d'entre elles à une augmentation de la fréquence des cancers.

La réparation des lésions d'un seul brin est rapide car l'autre brin, intact, sert de modèle. Celle des lésions des deux brins (cassure de la molécule d'ADN) est plus complexe car elle doit faire appel à l'autre molécule d'ADN de la paire. Ces mécanismes de recombinaison sont sujets à erreur. Il résulte un nombre appréciable de réparations fautives qui sont sources de lésions incompatibles avec la division cellulaire, ou de mutations. Quand les lésions sont graves un mécanisme d'apoptose (mort programmée) est déclenché qui tue la cellule lésée (Kahn et Briand, 1993). Seules les cellules porteuses de réparations fautives passées inaperçues, n'ayant pas provoqué d'apoptose, et compatibles avec la division cellulaire peuvent être à l'origine de cancers. Or le nombre de ces lésions est influencé par la dose et le débit de dose.

Aux faibles doses, ou débits de dose, le stock enzymatique est suffisant pour réparer les lésions, il y a peu de réparations fautives. Aux forts débits de dose les systèmes sont moins efficaces car ils doivent faire face à un grand nombre de lésions (Académie des Sciences, 1995). De plus aux débits de dose élevée on a observé chez les bactéries, et plus récemment dans les cellules de mammifères, l'apparition de systèmes de réparation, dits $S O S$, inductibles, impliqués dans les recombinaisons qui sont capables de réparer rapidement un grand nombre de lésions mais qui sont moins sûrs, d'où une augmentation de la proportion de réparations fautives (Académie des Sciences, 1995). Ces deux variations d'efficacité s'opposent à l'idée d'une extrapolation linéaire des fortes doses vers les faibles doses. Une discontinuité est vraisemblable (Académie des Sciences, 1995).

La prolifération compensatrice des cellules survivantes (Tubiana et al., 1992 ; Tubiana et al., 1990) est également en faveur d'une moindre efficacité des faibles doses. Depuis trente ans on sait que quelques heures après une irradiation les cellules du tissu irradié entrent en division cellulaire sous la stimulation de facteurs de croissance pour combler les vides provoqués par la mort cellulaire (Tubiana et al., 1990). Ce phénomène s'observe aussi bien dans des tumeurs (repopulation tumorale) que dans les tissus sains et peut être étudié 
avec une particulière netteté pour les cellules souches hématopoïétiques qui sont normalement quiescentes (hors cycle cellulaire, en $\mathrm{G}_{\mathrm{o}}$ ) et entrent en cycle de division une dizaine d'heures après une irradiation. Or dès les débuts de la radiobiologie (loi de Bergonié et Tribondeau, 1906) on a montré que les cellules qui ne se divisent pas sont beaucoup plus radiorésistantes que celles qui se multiplient, ce qui est, nous le savons maintenant, dû à une meilleure réparation des lésions de l'ADN dans les cellules quiescentes. Contrairement à ce qui survient à dose élevée, après une dose inférieure à $200 \mathrm{mSv}$, la mortalité cellulaire est insignifiante et ne provoque pas de prolifération compensatrice, le cycle est ralenti ou arrêté à cause de l'activation du gène $\mathrm{p} 53$ qui induit la sécrétion de protéines bloquant le cycle, ce qui favorise la réparation des lésions (Académie des Sciences, 1995). Aux plus fortes doses la sécrétion de facteurs de croissance surmonte ce blocage (Tubiana et al., 1992; Tubiana et al., 1990).

Or après irradiation, les divisions cellulaires, comme on pouvait théoriquement le prévoir, augmentent considérablement l'effet cancérogène, comme l'ont montré des données expérimentales in vivo et in vitro (Cohen, 1990; Gould, 1984 ; Kennedy et Little, 1984 ; Little, 1989) et des données épidémiologiques : ainsi l'irradiation du sein (Hrubec et al., 1989; Miller et al., 1989) ou de la thyroïde chez un adulte n'a pas d'effet cancérogène, sans doute parce que les cellules sont quiescentes, alors qu'à dose égale l'effet cancérogène chez le jeune enfant est notable et d'autant plus grand que l'enfant est plus jeune donc que les cellules prolifèrent plus rapidement (UNSCEAR, 1988, 1994; Walinder, 1995).

L'analyse des phénomènes radiobiologiques élémentaires apporte donc des arguments en faveur d'une moindre efficacité des faibles doses. Il en est de même pour les cancérogènes chimiques (Abelson, 1994) et de nombreux travaux montrent que les carcinogènes chimiques ne sont efficaces que quand l'administration du cancérogène est suivie de plusieurs divisions cellulaires, ce qui expliquerait l'absence d'effet cancérogène après l'administration de faibles doses de cancérogène chimique (Abelson, 1994), phénomène sur lequel de nombreux auteurs ont récemment insisté et qui s'oppose, pour ces produits, à l'extrapolation sans précaution, à partir des effets constatés après administration de fortes concentrations, vers la prédiction de l'effet cancérogène à faible concentration (Abelson, 1994 ; Cohen et Ellwein, 1990 ; Tubiana et al., 1992).

Quant au mécanisme en cause, les divisions cellulaires peuvent interférer avec la réparation de l'ADN et rendre irréversibles des lésions qui auraient été réparées en l'absence de division; la notion de "lésion potentielle", s'exprimant ou non selon les conditions dans lesquelles la cellule vit après irradiation, est d'ailleurs fondamentale en radiobiologie (Tubiana et al., 1990).

\subsection{Les mécanismes de cancérogenèse}

Dès le début des études sur la cancérogenèse, Berenblum (1947) avait montré l'existence de plusieurs étapes et introduit une distinction entre l'étape initiale (l'initiation) et les étapes ultérieures (promotion) dans lesquelles la multiplication cellulaire joue un rôle essentiel. 
Le modèle linéaire (ou linéaire quadratique) implique que pour les faibles doses le passage d'une seule particule à travers une seule cellule peut déterminer sa transformation cancéreuse, par lésion d'une seule molécule d'ADN. Sans analyser ici les mécanismes de cancérogenèse il faut rappeler que l'on estime que celle-ci requiert plusieurs lésions irréversibles du génome d'une cellule, en général 6 à 8 dans les épithéliomas, vraisemblablement moins dans certaines leucémies ou sarcomes mais toujours plusieurs. Même en admettant qu'une seule particule puisse déterminer une lésion irréversible; celle-ci ne pourrait, au pire, que constituer l'une des nombreuses étapes conduisant à la cancérisation. Pour qu'une cellule devienne cancéreuse au moins deux types de lésions sont nécessaires : 1) l'activation d'un ou plusieurs oncogènes. Celle-ci peut résulter d'une mutation ponctuelle et peut donc être causée par une seule particule car il s'agit d'un mutation dominante, 2) l'inactivation d'un gène suppresseur (appelé parfois anti-oncogène) qui est une étape indispensable. Cette inactivation nécessite la mutation ou la délétion des 2 gènes homologues (des deux allèles) (Weinberg, 1991). Il est donc inconcevable qu'elle puisse être déterminée par une seule particule et l'inactivation requiert l'altération de l'autre gène ou sa perte au cours de remaniements chromosomiques ultérieurs qui impliquent de nombreuses divisions cellulaires. 3) comme il est peu vraisemblable que tant d'altérations s'accumulent, simplement du fait du hasard, dans le génome d'une même cellule, on avait, depuis une décennie, postulé l'existence dans les cellules devenues cancéreuses d'une instabilité génétique. On a récemment découvert dans plusieurs types de cancers l'existence de gènes mutateurs (perturbant les systèmes de réparation de l'ADN) qui semblent constituer un troisième type de gène impliqué dans la cancérogenèse.

Etant donné les longs délais entre l'irradiation initiale et l'apparition des cancers (3 à 10 ans pour les leucémies, 10 à 40 ans pour les autres cancers) il est vraisemblable que l'irradiation joue un rôle d'initiation, c'est-à-dire cause l'une des toutes premières lésions du génome. Or les facteurs d'initiation (chimiques ou physiques), sont très nombreux et l'initiation n'est pas un facteur limitatif et ce sont plutôt les étapes ultérieures, celles de la promotion, qui paraissent déterminer la fréquence des cancers (Gould, 1984 ; Kennedy et Little, 1984).

Une autre hypothèse implicite du modèle de cancérogenèse utilisé par l'IRCP (1991) et de la relation linéaire ou linéaire quadratique est que l'irradiation d'une seule cellule suffit à provoquer un cancer indépendamment de l'état des cellules voisines. Cette hypothèse ne concorde pas avec ce que nous savons en radiobiologie et en cinétique cellulaire (Wallinder, 1995). Une cellule isolée est beaucoup plus radiosensible qu'une cellule ayant des relations normales (jonctions intercellulaires) avec les cellules voisines comme le montrent les expériences in vivo et in vitro avec les sphéroïdes (Tubiana et al., 1990). Ceci pourrait expliquer la plus grande sensibilité à la leucémogénèse puisque les cellules souches hématopoiétiques sont isolées. De plus les cellules saines exercent un contrôle sur la prolifération des cellules adjacentes et peuvent inhiber la prolifération d'une cellule initiée (Redpath et al., 1989 ; Tubiana et al., 1992). 
Au contraire la présence à côté de la cellule initiale de cellules lésées peut stimuler sa prolifération (Walinder, 1995). Ce problème a été abordé expérimentalement en comparant l'effet cancérogène d'une irradiation homogène (dans laquelle toutes les cellules d'un tissu reçoivent la même dose) ou hétérogène (par exemple après administration d'un radioélément émettant des particules de courte trajectoire). Si l'effet cancérogène était lié à la lésion d'une seule cellule, une irradiation hétérogène devrait, à dose moyenne égale, être plus efficace, certaines cellules recevant des doses plus élevées. Or c'est le contraire qui est observé (Académie des Sciences, 1995 ; Chmelevsky et al., 1984). Il est donc vraisemblable que les cellules voisines jouent un rôle important. L'hypothèse simplificatrice admettant que la transformation d'une seule cellule au sein d'un tissu normal suffit à provoquer un cancer apparaît donc discutable. D'ailleurs plusieurs données expérimentales prouvent le rôle des autres cellules; ainsi dans de nombreux cancers comme par exemple le cancer du sein on observe des anomalies dans le fonctionnement des cellules normales avoisinantes qui facilitent le développement du cancer (Chambon).

\subsection{L'hormesis, la réaction adaptive à de très faibles doses et l'effet des faibles doses}

Depuis plusieurs décennies de nombreux faits suggèrent que des faibles doses de radiations peuvent stimuler des systèmes enzymatiques, notamment de réparation et avoir un effet protecteur que l'on a appelé hormesis (Meyniel, 1995). En 1994 le rapport des Nations Unies (UNSCEAR, 1994) a consacré un chapitre à ce phénomène en discutant notamment les enquêtes qui suggèrent une diminution de la fréquence des cancers à faibles doses (Matanoski, 1987 ; Meyniel, 1995 ; Shimizu et al., 1987 ; UNSCEAR, 1994) (Fig. 8). A la suite de faibles doses (de l'ordre d'une dizaine de $\mathrm{mSv}$ délivrés à un débit supérieur à 0,2 Sv/minute) certains types de cellules (par exemple les lymphocytes) peuvent devenir plus résistantes (Meyniel, 1995 ; UNSCEAR, 1994). Sans insister sur ce phénomène nous en rapprocherons des données expérimentales qui suggèrent que des faibles doses de radiations pourraient non seulement ne pas diminuer la durée de la vie de souris ou de chiens mais l'allonger faiblement. Bien que les résultats des études sur différentes lignées de souris ne soient pas concordants il apparaît que pour des doses totales inférieures à $2 \mathrm{~Sv}$ délivrées en irradiation continue avec un débit de dose de $5 \mathrm{mSv}$ à $100 \mathrm{mSv} / \mathrm{j}$ il n'y a pas d'augmentation de la fréquence des cancers ni de réduction de la durée de vie (Lorenz, 1950 ; Pollycove, 1995 ; Wyngaarden, 1995). En irradiation aiguë rien, ou peu, n'est observé chez l'animal pour des doses inférieures à $200 \mathrm{mSv}$.

\section{Données épidémiologiques récentes}

Elles confortent pour l'essentiel les résultats de l'expérimentation animale. Rappelons les principales.

Chez les malades irradiés outre les données examinées plus haut qui montrent une diminution progressive du risque après des délais supérieurs à 15 ans, 
et un effet cancérogène et leucémogène inférieur à celui calculé en utilisant les coefficients de risque des sujets $\mathrm{H}+\mathrm{N}$, plusieurs résultats récents méritent mention. D'abord l'absence d'augmentation de la fréquence des cancers de la thyroïde et des leucémies chez les 47000 sujets examinés ou traités avec l'iode radioactif $\mathrm{I}^{131}$ (dose thyroïdienne moyenne environ $1,5 \mathrm{~Sv}$ ) alors que chez les sujets ayant reçu plus de $400 \mathrm{mCi}$ d'iode pour traitement d'un cancer thyroidien la fréquence des leucémies est augmentée, notamment chez les sujets ayant en plus reçu une irradiation externe (Hall et al., 1991, 1992 ; UNSCEAR, 1994). Ce risque est d'ailleurs inférieur à celui prédit par le coefficient de leucémogénèse de l'ICRP. Deux autres études effectuées chez des enfants traités pour angiome de la région cervicale pendant les premiers mois de la vie confirment l'extrême radiosensibilité de la thyroïde du jeune enfant et montrent une augmentation de la fréquence des cancers thyroïdiens (dose moyenne 1,1 Gy). Le coefficient de risque reste cependant inférieur à celui de $\mathrm{H}+\mathrm{N}$. La comparaison chez 400 enfants entre irradiation à débit élevé et à faible débit montre le rôle essentiel du débit (Académie des Sciences, 1995 ; De Vathaire, 1993 ; UNSCEAR, 1994).

Le nombre élevé des cancers du corps thyroïdien (environ 500) observé dans les environs de Tchernobyl à la suite de l'accident montre toutefois que des doses élevées (plusieurs Sv) délivrées par l'iode radioactif, iode 131 et iodes radioactifs à période plus courte, peuvent avoir un effet cancérogène important chez les jeunes enfants (UNSCEAR, 1994). L'interprétation de ces données doit tenir compte des très fortes doses d'irradiation dues notamment à la carence iodée qui a augmenté la fixation d'iode radioactif par la thyroïde. Il faut noter qu'avec le recul actuel, aucune augmentation de la fréquence des leucémies n'a été décelée autour de Tchnernobyl.

Plusieurs enquêtes n'ont pas décelé d'effet cancérogène. Ainsi celle sur les 65000 femmes canadiennes surveillées par radioscopies itératives pour pneumothorax thérapeutique, a montré une augmentation de la fréquence des cancers du sein, mais aucune augmentation de la fréquence des cancers du poumon ou des leucémies malgré des doses de $1 \mathrm{~Sv}$ au poumon (Howe, 1995). Corrélativement chez les mineurs des mines d'uranium on n'observe aucun excès de leucémies malgré une augmentation de la fréquence des cancers du poumon. Cependant dans ce cas il faut tenir compte de l'existence dans les mines de co-cancérigènes agissant sur le poumon (poussières, arsenic) (UNSCEAR, 1994).

Plusieurs études ont été effectuées en Russie sur des travailleurs de Mayak, la première usine nucléaire russe (The science of the total environment, 1994). Au début de l'activité de cette usine, de nombreux travailleurs ont reçu des doses de rayonnement externes supérieures à $1 \mathrm{~Sv}$ par an ; seule la mortalité par leucémies et lymphomes apparaît supérieure à celle de la population russe non irradiée alors que la mortalité par tumeur solide n'est pas augmentée. Une augmentation de la mortalité par cancer du poumon n'est observée que chez les travailleurs ayant manipulé du plutonium (UNSCEAR, 1994). Cette dissociation entre leucémies et tumeurs solides est semblable à celle qui a été observée dans certaines études expérimentales (Académie des Sciences, 1995). 
Pour les populations vivant dans la Vallée de la Técha (environs de Mayak) où des contaminations très importantes avaient été causées par les déchets, aucune augmentation de la fréquence des tumeurs solides n'est notée et celle de la fréquence des leucémies n'est observée qu'à partir de doses supérieures à $500 \mathrm{mSv}$ (Académie des Sciences, 1995 ; UNSCEAR, 1994).

En ce qui concerne l'irradiation des travailleurs en Occident, des données déjà anciennes sont assez rassurantes (Trott et Streffer, 1990). Chez les radiologistes qui exerçaient entre 1920 et 1939, la fréquence des leucémies était dix fois plus grande que chez les autres médecins, alors qu'elle est devenue semblable à celle des autres médecins à partir de 1950 (Matanoski et al., 1994), bien que les précautions prises alors, sur la base de normes de protection bien moins sévères que celles en vigueur aujourd'hui sembleraient très insuffisantes. L'étude récente la plus importante est celle du CIRC portant sur 95000 travailleurs de l'industrie nucléaire des Etats-Unis, de Grande-Bretagne et du Canada (Cardis et al., 1995 ; IARC, 1994). Elle ne décèle aucune augmentation de la fréquence des cancers - sauf des leucémies - bien que parmi les travailleurs environ 2000 aient reçu une dose supérieure à $400 \mathrm{mSv}$ étalée sur leur existence professionnelle. Le risque relatif de cancer est même légèrement inférieur à la normale. Cependant, en raison d'un grand intervalle de confiance, cette estimation négative ne permet pas de rejeter le coefficient de risque cancérogène de l'IRCP, bien qu'aucun des 21 cancers en excès prévus par le coefficient de risque, avec le suivi actuel qui est supérieur à 40 ans, ne se soit produit. En revanche un excès de leucémies a été observé, notamment à l'usine de Sellafield où ont été observées 4 des 6 leucémies survenues chez les travailleurs ayant reçu des doses supérieures à $400 \mathrm{mSv}$; ce risque leucémogène est compatible avec la prédiction du coefficient de l'ICRP 60 qui est de 9. Rappelons par ailleurs que l'établissement de Sellafield est particulier, puisque dans ce village on a observé une élévation de ce type de leucémies chez les enfants dont on a montré qu'elle n'était pas liée à une pollution radioactive (Doll et al., 1994). Lorsque le suivi de la cohorte sera achevé, l'estimation du risque prévoit 191 cancers dont 28 leucémies, le coefficient de risque antérieur (Koshland, 1994) prédisait 38 cancers dont 5 leucémies. Le coefficient de risque de l'ICRP 60 (1991) apparaît donc excessif pour les cancers solides alors que celui de l'ICRP 26 (1977) pourrait être un peu faible pour les leucémies. L'étude du CIRC sera complétée dans les années à venir notamment grậce à l'inclusion des travailleurs français pour lesquels les données devraient bientôt être disponibles. Cette augmentation de puissance statistique devrait permettre de déterminer, de façon statistiquement significative, si le coefficient de risque estimé par l'ICRP 60 doit, ou non, être rejeté. Alors qu'il y a quelques années encore on croyait impossible de pouvoir obtenir par des études épidémiologiques des données valables sur l'effet des faibles doses, ce résultat semble maintenant à portée de la main.

Dans une étude qui a porté sur plus de 100000 travailleurs des arsenaux américains dont 28000 étaient exposés à des doses supérieures à $5 \mathrm{mSv}$, on n'a observé aucune augmentation de la fréquence des cancers ou des leucémies 
malgré un recul de 45 ans et un grand nombre de cas de cancers (Académie des Sciences, 1995 ; UNSCEAR, 1994). Cette absence d'effet malgré une dose collective d'environ $1500 \mathrm{~h}$ Sv suggère que l'efficacité des faibles doses est très petite. On a observé une augmentation de la fréquence des mésothéliomes vraisemblablement due à l'amiante, alors que pour les autres cancers le risque est inférieur à celui des ouvriers non exposés aux radiations.

Une autre source importante d'information sur les faibles doses provient de l'étude des populations soumises à différents niveaux d'irradiation naturelle. Celle-ci varie à la surface de la terre, entre 1,5 et $6 \mathrm{mSv} / \mathrm{an}$, exceptionnellement elle peut dépasser $15 \mathrm{mSv} / a n$ (UNSCEAR, 1988). Aucune augmentation de la fréquence des cancers n'a été décelée (Académie des Sciences, 1989; Luxin et al., 1990 ; Nambi et Soman, 1987 ; UNSCEAR, 1994 ; Wang, 1993 ; Wang et al., 1990). Plusieurs études ont comparé la fréquence des leucémies dans des régions à haute et basse radioactivité naturelle. Il n'existe en Grande Bretagne ou en France aucune élévation de la fréquence des leucémies dans les régions à forte irradiation naturelle (Muirhead, 1991). Une enquête effectuée dans deux régions de la Chine où les doses annuelles d'irradiation à la moelle osseuse sont respectivement de $1,9 \mathrm{mSv}$ et $0,72 \mathrm{mSv}$ a montré que la fréquence des leucémies était environ $30 \%$ plus faible dans la région où la dose était la plus forte (Wang et al., 1990). Or le calcul théorique prévoyait que pour une telle différence de dose (60 mSv en 50 ans), le risque aurait dû être augmenté d'environ $20 \%$. Une autre étude effectuée en Chine a analysé l'influence de l'irradiation naturelle sur la fréquence des nodules et cancers thyroïdiens : pour une différence de dose de $100 \mathrm{mSv}$ en 50 ans, aucune différence n'a été constatée (Wang et al., 1990). Cependant dans toutes ces enquête la puissance statistique est trop faible pour permettre d'exclure le coefficient de risque proposé par l'ICRP.

L'irradiation naturelle la plus importante et la plus variable selon les régions est celle due au radon. Malgré une vingtaine d'enquêtes épidémiologiques, aucun excès significatif de la fréquence des cancers du poumon n'a été décelé dans les régions à forte concentration en radon, bien que l'irradiation pulmonaire supplémentaire ait pu atteindre plusieurs centaines de mSv (Cohen, 1995 ; Doucet et Jammet, 1985 ; UNSCEAR, 1994). Ces enquêtes géographiques sont cependant critiquables dans la mesure où les doses individuelles ne sont pas connues et une association entre exposition moyenne et incidence pourrait être masquée par d'autres facteurs, notamment le tabac. Des études cas-témoin ont été effectuées, dans lesquelles les doses reçues par chaque individu ont été estimées ; on a pu ainsi comparer la dose pulmonaire des sujets atteints de cancer du poumon et des témoins. Les résultats discordants de ces études ne permettent ni de confirmer ni d'exclure formellement un effet cancérogène du radon (Académie des Sciences, 1995 ; UNSCEAR, 1994). Une étude récente a le mérite d'éliminer le facteur tabac en s'adressant uniquement à des femmes n'ayant jamais fumé; elle n'a décelé aucune influence de la concentration en radon dans les habitations sur l'incidence du cancer du poumon (Alavanja et al., 1994). Ainsi ces enquêtes n'apportent 
aucun argument en faveur du rôle du radon dans la genèse des cancers du poumon mais, en l'absence d'une puissance statistique suffisante, elles ne permettent pas de l'exclure. Il est donc souhaitable que ces études soient poursuivies et regroupées (Académie des Sciences, 1995). Il apparaît en tous cas qu'il n'est pas justifié d'estimer le risque du radon dans les habitations par une extrapolation linéaire effectuée à partir des observations faites dans les mines d'uranium ou bien d'autres facteurs cancérogènes sont présents (arsenic, poussière). D'ailleurs le peu de crédibilité du rôle du radon domestique dans la genèse des cancers du poumon est souligné par l'absence de volonté des gouvernements pour agir dans ces domaines.

Enfin des enquêtes effectuées sur des sujets vivant au voisinage des établissements nucléaires aux Etats-Unis et en France n'ont révélé aucune augmentation de la fréquence des leucémies ou des cancers mais les doses reçues étaient minimes et ce résultat négatif n'est pas surprenant (Hill et Laplanche, 1990 ; Jablon et al., 1990). L'excès de leucémies observé au Royaume-Uni au voisinage des établissements de Sellafield et de Dounreay ne semble pas en relation avec une irradiation ou une pollution radioactive; les conclusions des récents articles (Doll et al., 1994) et ceux de l'enquête effectuée à l'occasion du procès rejettent l'hypothèse d'un rôle causal de l'irradiation des enfants ou des pères.

Les deux rapports de l'Académie des Sciences $(1989,1195)$ n'ont pas pris en compte pour l'évaluation des effets cancérogènes des faibles doses, les résultats des enquêtes sur les irradiations in utero, suivant en cela la position du rapport de l'UNSCEAR (1986) dans lequel il était dit : "le Comité a décidé d'accepter avec réserve la nature causale de la relation entre l'effet cancérogène et l'irradiation in utero tout en soulignant que c'est simplement par prudence et non sur des données scientifiques fermement établies". En 1994 le rapport de l'UNSCEAR (1994) déclarait : "les études sur les expositions in utero ont fourni une gamme étendue du coefficient de risque depuis un risque relativement élevé jusqu'à un risque très faible et indétectable, incluant un risque nul. Comme il n'y a pas de raison biologique de penser que l'embryon et le fœtus sont résistants aux effets de rayonnements et en particulier à la radiocancérogenèse, l'observation d'un effet cancérogène appréciable doit être attendue. Cependant toute tentation de quantification du risque fondée sur les données actuellement disponibles est sujette à une grande incertitude".

Il est peu vraisemblable que ce problème soit élucidé car les examens radiologiques in utero ont maintenant été remplacés par l'échographie et les expérimentations animales sont restées négatives, peut-être en raison d'un nombre insuffisant d'animaux d'expérience.

En terminant revenons sur l'existence éventuelle d'un seuil.

Ce problème avait été envisagé dans le rapport 1989 (Académie des Sciences, 1989) mais il reste d'actualité (UNSCEAR, 1994). Le principal argument contre l'existence d'un seuil est qu'une irradiation même faible peut causer des lésions irréversibles de l'ADN et que celles-ci peuvent contribuer à 
un processus de cancérisation en ajoutant une lésion supplémentaire à celles dues à d'autres causes. Cet argument postule que les lésions de l'ADN provoquées par les rayonnements ionisants pourraient être moins réparables que celles dues aux agents d'oxydation normalement présents dans les cellules, car les transferts d'énergie élevés qui peuvent survenir causent des lésions irréparables. D'après cet argument les rayonnement ionisants provoqueraient des lésions différentes de celles causées par le métabolisme normal dans la cellule ou par les cancérogènes chimiques. Cependant cette assertion n'a pas été étayée par des données expérimentales. Or parmi les rayonnements ionisants, les transferts d'énergie linéaire les plus élevés sont ceux causés par les particules alpha et ce sont donc pour elles que la proportion de lésions irréparables de l'ADN devrait être la plus forte. Or dans le cas d'irradiation par particules alpha, tant chez l'animal (Raabe, 1984 ; Raabe et al., 1980) que chez l'homme les données suggèrent fortement l'existence d'un seuil (Cohen, 1995 ; Rowland et Stehney, 1983). La figure 11 montre la relation dose-effet chez les peintres en cadrans lumineux contaminés par le radium 228 et les figures 12 et 13 correspondent aux hépatomes chez les sujets ayant reçu du thorotrast pour examen radiologique (UNSCEAR, 1994). Dans les deux cas on n'observe un excès de cancers que pour des doses supérieures à quelques Sv. Comme l'a conclu le rapport BEIR IV (Committee on biological effects, 1988) consacré aux irradiations particulaires, l'existence d'un seuil pratique est extrêmement vraisemblable dans ces cas. Comme le délai de latence est d'autant plus long que la dose est plus faible, le seuil pourrait être du à ce que le délai devient supérieur à l'espérance de vie. Ces résultats ne sont pas compatibles avec le modèie de l'ICRP puisque c'est pour ce type de rayonnement que la relation linéaire sans seuil à partir des doses les plus basses devrait être la plus plausible.

Enfin il est impossible de discuter de cancérogenèse par agents physiques sans la comparer avec celle par agent chimiques. De nombreux travaux ont montré dans ce cas que l'extrapolation linéaire sans seuil à partir des effets observés aux concentrations élevées vers les faibles concentrations aboutirait à une importante surestimation notable du risque (Abelson, 1994 ; Tubiana et al., 1992). 


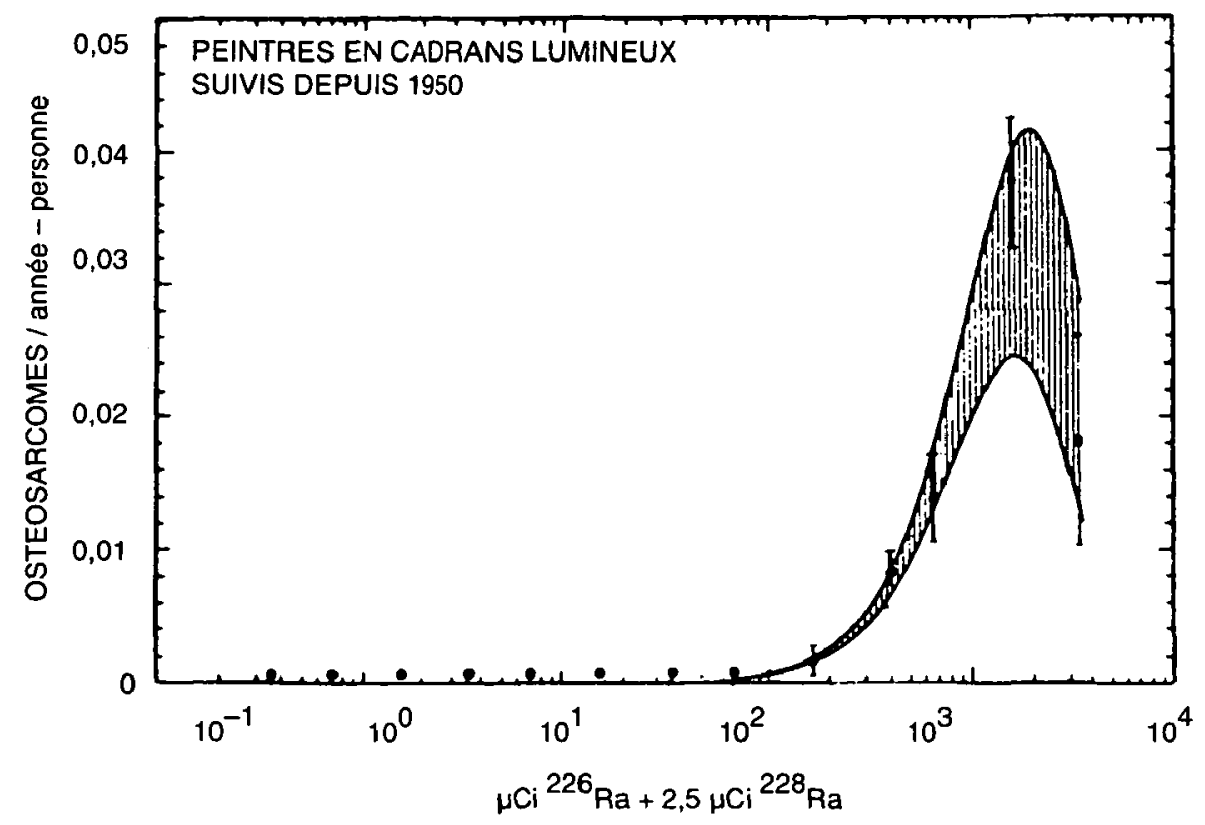

Fig. 11 - Relation dose-effet pour les ostéosarcomes provoqués par une irradiation interne par le Radium 226 + 228 (émetteur $\alpha$ de 1600 ans de période) chez les peintres en cadrans lumineux. Aucune augmentation de la fréquence des cancers n'est observée pour des activités faibles. A partir d'une activité correspondant à une irradiation totale de quelques grays, la fréquence (exprimée en nombre de cas par an par personne exposée au risque) croît d'abord très lentement puis rapidement, passe par un maximum pour quelques dizaines de grays, puis décroît. La fonction qui décrit le mieux cette relation dose-effet est $: \mathrm{I}=\left(\mathbf{c}+\beta \mathrm{D}^{2}\right) \mathrm{e}^{-\gamma \mathrm{D}}$ où $\mathrm{D}$ est la quantité de radium fixée. La zone grisée indique la bande couverte par la fonction. Les barres d'erreur correspondent à l'erreur standard. (D'après Rowland, Stehney, Lucas, 1983). (Dose-response relationship for radium-induced bone sarcomas, Health Phys., 44 Suppl. 1, 15-31).

Dose-effect relation for osteosarcomas in luminous dial painters caused by internal irradiation by radium $226+228$ ( $\alpha$-emitter with half-life 1600 years). No increase in incidence of cancer was seen with low doses. Above an activity corresponding to a dose of a few grays, the incidence (expressed as the number of cases per year per person exposed) increases at first very slowly, then more rapidly and passes through a maximum for several tens of gray and then decreases. A function which fits this dose-effect relation is $\mathrm{I}=\left(\mathrm{c}+\beta^{2}\right) e^{-\gamma \mathrm{D}}$ where $\mathrm{D}$ is the quantity of radium fixed. The hatched area shows the band covered by the function. Error bars correspond to standard errors. (After Rowland, Stehney, Lucas, 1983). (Dose-response relationship for radium-induced bone sarcomas, Health Phys., 44 Suppl. 1, 15-31.) 


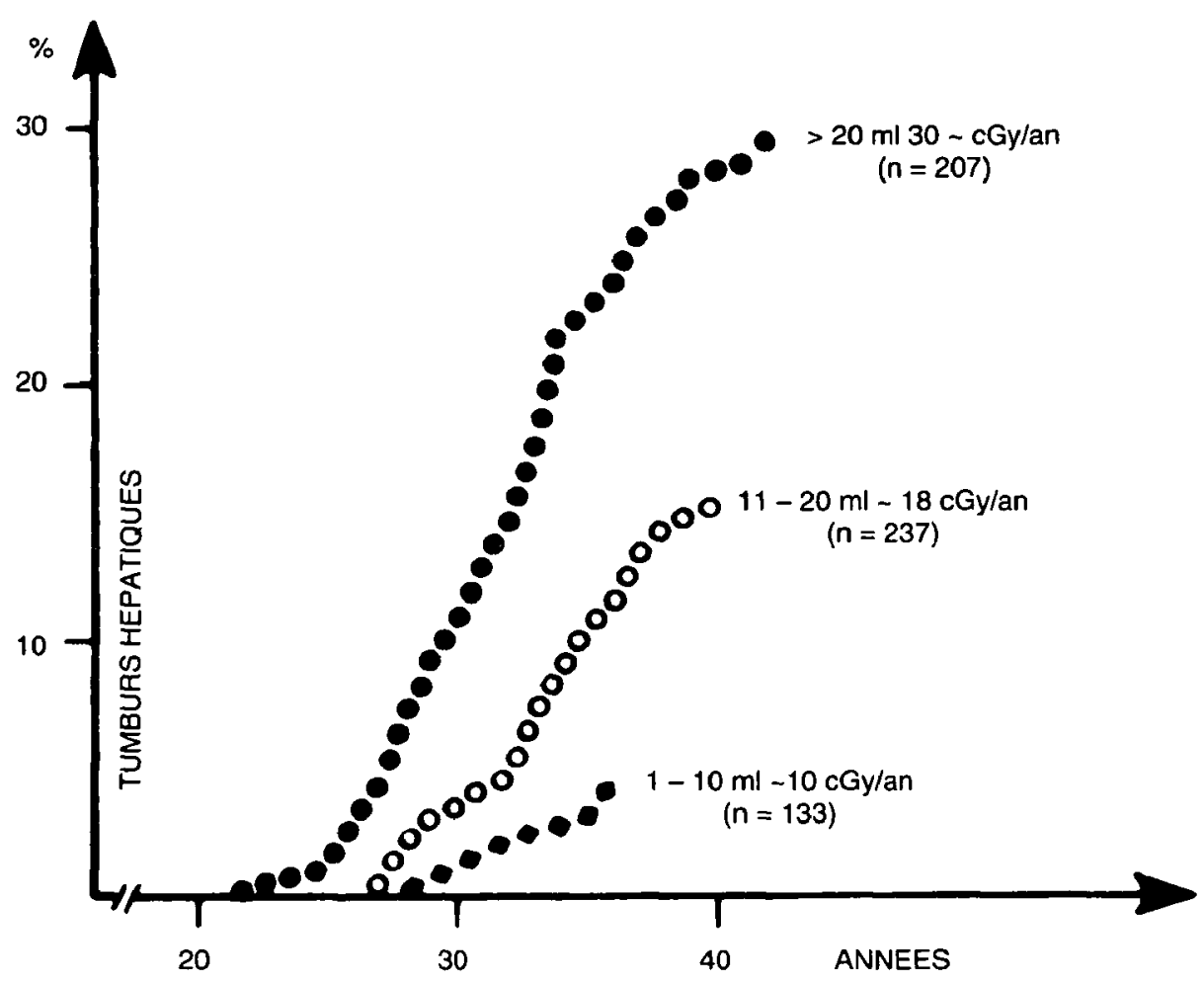

Fig. 12 - Hépatomes provoqués chez l'homme par l'injection de dioxide de Thorium $\left(\mathrm{ThO}_{2}\right)$, ou thorotrast, produit de contraste autrefois utilisé en radiologie vasculaire. Le débit de dose pour cet émetteur $\alpha$ est très hétérogène en raison du caractère de sa fixation par les cellules du parenchyme hépatique. Parmi 2135 sujets et sur 1689 décès, 256 cas de cancers hépatiques ont été observés de 16 à 40 ans après injection. Plus la dose est grande, plus la fréquence des hépatomes est élevée et plus le délai d'apparition est court. On observe également un délai d'autant plus long que la dose est plus faible après contamination par un cancérogène chimique. Ces données suggèrent l'existence d'un seuil pratique quand le délai d'apparition devient supérieur à l'espérance de vie du sujet irradié. (D'après G. von Kaick et al. (1984), Radiation carcinogenesis, pp. 253-262, Raven Press.)

Hepatomas in man caused by injection of thorium dioxide or thorotrast, a contrast medium once used in vascular radiology. The dose rate due to this $\alpha$ emitter is very heterogeneous owing to its fixation by the parenchyma of the liver. Out of 1689 deaths in 2135 subjects there were 256 cases of liver cancer 1640 years after injection of thorotrast, the total dose being between 2 and 15 Gy. The figure shows that the incidence of hepatomas is greater with larger doses. Also there seems to be a slightly shorter latent period with high doses. (After G. von Kaick et al. (1984), Radiation carcinogenesis, pp. 253-262, Raven Press.) 


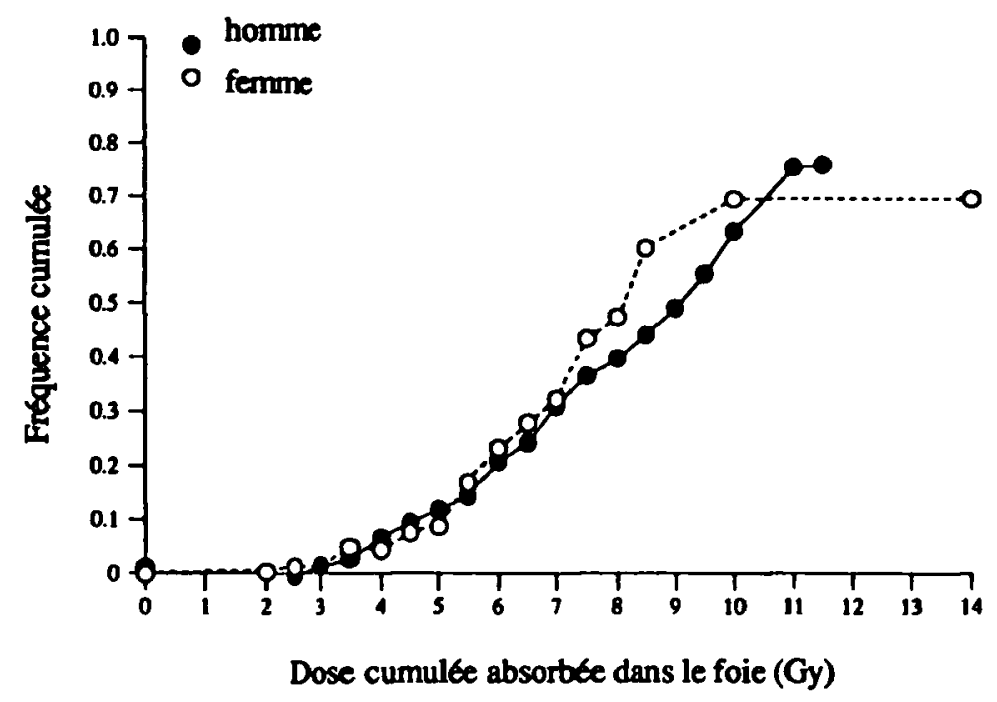

Fig. 13 - Incidence cumulative des cancers du foie après injection du thorotrast (étude danoise). Aucune augmentation dans la fréquence de ces cancers n'est observée pour des doses inférieures à $3 \mathrm{~Gy}$ (d'après le rapport UNSCEAR, 1994).

Cumulative incidence of liver cancers after injection of thorotrast (Danish study). No increase in the incidence of these cancers was observed for doses lower than 3 Gy (after the UNSCEAR, 1994 report).

\section{Conclusion}

Deux récents éditoriaux du prestigieux journal américain "Science", concluent à l'inadéquation du modèle linéaire sans seuil. Abelson (1994) conclut ainsi son éditorial du 9 septembre : "La façon dont on extrapole des effets des fortes doses vers les effets des faibles doses est erronée tant en ce qui concerne les produits chimiques que les radiations. Des niveaux d'exposition sans risque existent. Le public a été sans raison effrayé et trompé et des centaines de milliards de dollars gaspillés". Il met en doute l'assertion selon laquelle une seule molécule endommagée peut causer un cancer car cette assertion ignore les capacités de réparation de l'ADN. Il rappelle qu'après administration de faibles doses de produits chimiques les lésions de l'ADN sont moindres chez les animaux d'expérience que chez les animaux témoins.

Quelques mois plus tard Koshland (1994a, b) revient dans un autre éditorial de "Science" sur le même sujet et il écrit "Les nouvelles connaissances sur les mécanismes de réparation de l'ADN pourraient conduire à réexaminer le postulat de l'extrapolation linéaire pour les pesticides et les radiations et permettre une évaluation plus réaliste des risques environnementaux, basée non sur des opinions de protagonistes partisans mais de bons et solides faits scientifiques". C'est de toute évidence le but que nous devrions tous avoir. Il faut remarquer que nombreux radiobiologistes prestigieux ont exprimé des points de vue semblables (Thiessen, 1991 ; Walinder, 1995). 
On pourrait résumer ce qui précède en disant que rien ne subsiste des fondements théoriques et expérimentaux du modèle linéaire quadratique et $a$ fortiori de la simple extrapolation linéaire pour estimer l'effet cancérogène des doses inférieures à $200 \mathrm{mSv}$. Plusieurs données expérimentales sur la réparation de l'ADN et sur la cancérogenèse donnent à penser que la probabilité de l'induction d'un cancer pour de faibles doses est petite et que le rôle du débit de dose est crucial (Académie des Sciences, 1995; Latarjet, 1995). Il devrait donc exister une discontinuité entre les doses capables de modifier la cinétique cellulaire et les mécanismes de réparation de l'ADN et les doses, ou débits de dose, trop petits pour induire ces effets.

Il existe au sujet de l'estimation des effets cancérogènes des faibles doses deux positions extrêmes. Pour les uns, tenants du rapport de l'ICRP, les coefficients cancérogènes calculés en 1990 doivent rester intangibles tant que leur non validité n'aura pas été prouvée et toute discussion sur leur valeur constitue une atteinte à un dogme. Pour les autres au contraire il existe suffisamment de présomptions expérimentales et théoriques pour admettre l'existence d'un seuil, voire même d'un effet bénéfique des très faibles doses et pour eux le seul problème serait de déterminer son niveau. La position de l'Académie des Sciences exposée dans les rapports de 1989 et 1995 est située à mi-chemin entre ces deux attitudes. Elle constate que les données sur lesquelles on s'était appuyé en 1989 pour remettre en cause le coefficient de risque cancérogène à faible dose accepté jusque là, sont discutables et qu'il vaut mieux s'en tenir à ces valeurs tant que des données épidémiologiques ou expérimentales plus robustes n'auront pas permis de réduire l'incertitude actuelle. Par prudence, comme certaines données suggèrent que même délivrée avec un faible débit de dose, une dose cumulée atteignant quelques Sievert peut avoir un effet câncérogène, l'Académie des Sciences avait proposé en 1989, et a réaffirmé cette position en 1995, d'assortir la norme précédente $(50 \mathrm{mSv} / \mathrm{an}$ pour les travailleurs) d'une limite dose-vie cumulée posée égale à $1 \mathrm{~Sv}$, en prenant toutes les précautions utiles pour que cette dose-vie soit délivrée pendant un délai suffisamment long (20 ans), afin d'éviter que les travailleurs ne puissent être exclus d'une activité exposant à une irradiation pendant les dernières années de leur vie professionnelle.

Pour le public la dose limite de $1 \mathrm{mSv} / \mathrm{an}$ proposée par l'ICRP est plus discutable encore. Le calcul des risques causés par cette dose est basé sur tant d'hypothèses contestables qu'il n'a guère de signification. Rappelons qu'il est estimé à partir de l'effet cancérogène évalué pour des doses de 500 à $1000 \mathrm{mSv} / \mathrm{an}$. Le facteur d'extrapolation est donc de 500 à 1000 , c'est comme si, à partir du risque mesuré chez des sujets consommant 0,5 à 1 litre de vin par jour on voulait calculer le risque d'un baba au rhum par semaine. or si $1 \mathrm{mSv}$ par an entraînait un risque il devrait y avoir une différence dans la fréquence des cancers entre les régions à faible dose de rayonnement naturel et à forte dose puisque l'écart des doses dans ces cas est supérieure à $2 \mathrm{mSv}$. Or rien n'a été constaté. Certes il faut poursuivre et affiner ces études, mais est-il légitime d'ici là et sans aucun fondement sérieux d'affoler les populations vivant dans le 
Massif Central ou en Bretagne en fonction d'hypothèses très incertaines. Dire que cette limite est indiquée pour les rayonnements d'origine humaine et qu'il s'agit dans ces cas d'irradiation d'origine naturelle est un contresens scientifique. Un $\mathrm{mSv}$ est un $\mathrm{mSv}$ quelle que soit son origine et dans les deux cas il entraîne la même conséquence biologique (ou la même absence de conséquence).

Ces considérations soulignent l'absurdité des calculs estimant le nombre de cancers provoqués par des doses de quelques mSv/an, calculs qui ont fait la une des journaux après l'accident de Tchernobyl et ont été contredits depuis par les observations puisqu'aucune augmentation de la fréquence des leucémies n'a été observée. L'augmentation de la fréquence des cancers de la thyroïde s'explique aisément puisque les quantités d'iode radioactifs fixées dans la thyroïde des enfants ont été très élevées, aucune prévention par administration d'iode stable n'ayant été mise en œuvre.

Ces conclusions sont fondées sur les données radiobiologiques et épidémiologiques. Il appartient aux autorités politiques de juger de l'opportunité, ou non, d'accepter les normes proposées en tenant compte d'autres considérations. D'ailleurs une petite minorité au sein du groupe de travail de l'Académie a préconisé pour des raisons d'opportunité, un alignement sur la position des autres pays de l'Union Européenne.

Pour donner la dimension réelle du problème il est utile en terminant de donner quelques renseignements sur les doses effectivement reçues par les travailleurs du nucléaire et des services de radiologie des hôpitaux. Les valeurs moyennes annuelles d'exposition pour l'homme sont les suivantes (UNSCEAR, 1988) :

- Exposition externe d'origine naturelle :

- Exposition interne d'origine naturelle :

$\left.\begin{array}{l}0,77 \mathrm{mSv} \\ 1,56 \mathrm{mSv}\end{array}\right\} 2,3$

- Exposition d'origine médicale :

$1,00 \mathrm{mSv}$

- Industrie nucléaire (de l'extraction de l'uranium aux déchets) : $\quad 0,02 \mathrm{mSv}$

- Essais nucléaires atmosphériques :

$0,1 \mathrm{mSv}$

En France, environ 200000 travailleurs (dont plus de la moitié dans les hôpitaux) sont exposés aux rayonnements ionisants et sont surveillés par dosimétrie. L'analyse des résultats dosimétriques du Service Central de Protection Contre les Rayonnements Ionisants (SCPRI) de 1990 à 1993 montre que moins de $3 \%$ des travailleurs ont reçu une dose supérieure à $10 \mathrm{mSv} / \mathrm{an}$ et environ $1 \%$ plus de $15 \mathrm{mSv} / \mathrm{an}$. En ce qui concerne la dose cumulée pendant une existence professionnelle, les données du Centre International de Recherche sur le Cancer (CIRC) sur les 100000 travailleurs de l'industrie nucléaire des EtatsUnis, Royaume-Uni, Canada ayant régulièrement été suivis par dosimétrie indiquent que la dose-vie cumulée a été supérieure à $500 \mathrm{mSv}$ chez moins de $1 \%$ des travailleurs et supérieure à $1000 \mathrm{mSv}$ chez moins de 1 pour mille des 
travailleurs. Ces chiffres incluent les personnes ayant travaillé pendant les années 50, période où la radioprotection était balbutiante. Depuis 1960, dans tous les pays les doses ont été notablement réduites et si l'on se limitait aux travailleurs ayant commencé leur activité après 1960, les doses seraient encore plus faibles. Un abaissement des normes ne changerait donc rien à l'état actuel des choses.

En 1993, en France, la dose collective due à l'exposition de l'ensemble des travailleurs a été de $200 \mathrm{~h} \mathrm{~Sv}$ à comparer aux $50000 \mathrm{~h} \mathrm{~Sv}$ dus à l'irradiation médicale et aux $130000 \mathrm{~h}$ Sv liés à l'irradiation naturelle (Académie des Sciences, 1995). En effet, l'irradiation naturelle délivre une dose d'environ 2,5 mSv/an à l'ensemble de la population et l'irradiation médicale une dose moyenne de $1 \mathrm{mSv} / \mathrm{an}$ à l'ensemble de la population avec de grandes variations individuelles. Rappelons que les examens radiologiques délivrent des doses variant entre $0,1 \mathrm{mSv}$ et $200 \mathrm{mSv}$ selon le type d'examen. Or, des progrès réalisés dans les générateurs de rayons $\mathrm{X}$ ou dans les détecteurs seraient susceptibles d'entraîner une réduction notable de l'irradiation au cours de ces examens (Académie des Sciences, 1995). Logiquement, priorité devrait donc.être donnée à cette question sur le plan de la radioprotection.

\section{Résumé}

L'analyse des données radiobiologiques récentes, notamment celles concernant les systèmes de réparation de l'ADN et celles sur la carcinogenèse, conduit à penser que la relation linéaire quadratique ne peut pas être valablement utilisée pour estimer les risques de doses inférieures à $400 \mathrm{mSv}$.

De plus la relation linéaire quadratique est en opposition avec l'existence de la réduction dans l'effet cancérogène qui est observée quand le débit de dose diminue dans le domaine des faibles doses. Elle est de plus inconciliable avec les données obtenues après irradiation avec des particules alpha. Ainsi, si l'on ne peut pas exclure l'éventualité d'un effet cancérogène après de faibles doses, les données radiobiologiques disponibles suggèrent une discontinuité dans la relation dose-effet quand on tombe au-dessous de doses capables de provoquer un nombre notable de morts cellulaires (ce qui induit une prolifération cellulaire interférant avec la réparation cellulaire) ou un dysfonctionnement du système de réparation de l'ADN soit environ 200 à $500 \mathrm{mSv}$. Ceci souligne la nécessité de poursuivre les études épidémiologiques dans le domaine des faibles doses.

Aucun effet n'a jamais été observé chez l'adolescent ou l'homme adulte pour les doses inférieures à $200 \mathrm{mSv}$. L'ensemble des données épidémiologiques est donc en accord avec l'hypothèse d'une réduction de l'effet cancérogène pour les doses inférieures à environ $0,5 \mathrm{mSv}$ et du rôle prédominant dans la radiocancérogenèse de la prolifération cellulaire après irradiation. 


\section{RÉFÉRENCES}

Académie des Sciences, (1988) Cancérogenèse par les faibles doses de radiations ionisantes et normes de sécurité, 51 p. (R. Latarjet Ed.) Académie des Sciences, Paris.

Académie des Sciences (1989) Risques des rayonnements ionisants et normes de radioprotections : rapport 23 de l'Académie des Sciences. 72 p. Académie des Sciences, Paris.

Académie des Sciences (1995) Problèmes liés aux faibles doses des rayonnements ionisants : rapport $\mathrm{n}^{\circ} 34,121$ pages.

Abelson P.H. (1994) Risk assessments of low level exposures : éditorial. Science 265, 1507.

Alavanja M.C.R., Brownson R.C., Lubin J.H., Berger E., Chang J., Boice J.D. (1994) Residential radon exposure and lung cancer among nonsmoking women. J. Nat. Cancer inst. 86, 1829-1837.

Berenblum I., Shubik P. (1947) The role of croton oil applications, associated with a single painting of a carninogen in tumour induction of the mouse skin. British J. Cancer, 1, 379382.

Boice J.D., Blettner M., Kleinerman R.A., et al. (1987) Radiation dose and leukemia risk in patients treated for cancer of the cervix : J. Nat. Cancer Inst. 79, 1295-1311.

Cardis E., Gilbert E.S., Carpenter L. et al. (1995) Effects of low doses and low dose rates of external ionizing radiation : cancer mortality among nuclear industry workers in three countries. Radiat. Res. 142, 117-132.

Chau N.P. (1987) Radiation carcinogenesis in humans : is it necessary to revise exposure dose limits based on recent estimates of lifetime risk? Health Phys. 52, 753-761.

Chmelevsky D., Kellerer A.M., Lafuma J., Morin M. and Masse R. (1984) Comparison of the induction of pulmonary neoplasm in sprague-dawley rats by fission neutrons and radon daughters. Radiat. Res. 98, 519-535.

Cleaver J.E. (1994) It was a very good year for DNA repair. Cell. 76, 1-4.

Cohen B. (1995) Test of the linear-no threshold theory of radiation carcinogenesis for inhaled radon decay products. Health Phys. 68, 157-174.

Cohen S.M. \& Ellwein L.B. (1990) Cell proliferation in carcinogenesis. Science 249, 503504.

Committee on Biological Effects of Ionizing Radiation (BEIR IV) (1988) Health risks of radon and other internally deposited alpha-emitters, United States National Academy of Sciences, National Research Council. National Academic Press, Washington.

Curtis E. Boice J.D., Stovall M. et al. (1994) Relationship of leukemia risk to radiation dose following cancer of uterine corpus. J. Nat. Cancer Inst. 86, 1315-1324.

Darby S.C., Doll R., Gill SK. \& Smith P.G. (1987) Long term mortality after a single treatment course with $X$ rays in patients treated for ankylosing spondilitis. British J. Cancer 55, 179-190.

De Vathaire F., Fragu P. François P. (1993) Long term effect on the thyroid of irradiation for skin angiomas in chilhood. Radiat Res. 133, 381-386.

De Vathaire F., Shamsalfin A., Grimaus E. et al. (1995) Solid malignant neoplasms after childhood irradiation: Decrease of the relative risk with time after irradiation. C.R. Acad. Sc. Paris 318, 483-490.

Doll R., Evans H.J., Darby S.C. (1994) Paternal exposure not to blame. Nature 367, 678680.

Dousset M. \& Jammet H. (1985) Comparaison de la mortalité par cancer dans le Limousin et le Poitou-Charentes. Radioprotection 20, 61-67.

Goodhead D.T., Thadrer J. and Cox R. (Weiss Lecture) (1993) Effects of radiation of different qualities on cells : molecular mechanisms of dammage and repair. Int. J. Rad. Biol. 63, 543-546. 


\section{EFFETS DES FAIBLES DOSES}

Goodhead D.T. (1994) Initial events in the cellular effects of ionizing radiations : clustered damage in DNA. Int J. Rad. Biol. 67, 7-12.

Gould M.N. (1984) Radiation initiation of carcinogenesis in vivo : a rare or common cellular event. In : Radiation Carcinogenesis : Epidemiology and Biological Significance. J.D. Boice Jr and J.F. Fraumeni, Jr Eds. pp. 347-358 Raven Press, New-York.

Hall P. et al. (1992) Leukaemia incidence after iodine exposure. Lancet 340, 1-4.

Hall P., Holm L.E., Lundell G. (1991) Cancer risks in thyroid cancer patients. Brit. J. Cancer 64, 159-163.

Hill C., Laplanche A. (1990) Overall mortality and cancer mortality around French nuclear sites. Nature 347, 755-757.

Howe G.R. (1995) Lung cancer mortality between 1950 and 1987 after exposure to fractionated moderate-dose-rate ionizing radiation in the canadian fluoroscopy cohort study and a comparison with lung cancer mortality in the atomic bomb survivors study. Rad. Res. 142, 295-304.

Hrubec C.Z., Boice J.D., Monson R.R. \& Rosenstein M. (1989) Breast cancer after multiple chest fluoroscopies. Cancer Res. 49, 229-234.

I.A.R.C (1994) Study Group on Cancer Risk among Nuclear Industry Workers, Direct estimates of cancer mortality due to low dose of ionising radiations : an international study. Lancet 344, 1039-1043.

International Commission on Radiological Protection (ICRP) (1977) Recommendations of the ICRP (publication 26) Ann. ICRP 1.

International Commission on Radiological Protection (ICRP) (1991) Recommendations of the commission (publication 60) Ann. ICRP 21, 1-3.

Jablon S., Hrubec Z., Boice J.D. \& Stone B.J. (1990) Cancer in populations living near nuclear facilities. NIH publ., $\mathrm{n}^{\circ} 90874$.

Kahn A. et Briand P. (1993) L'apoptose, une mort programmée ou une prolifération avortée? Médecine/Sciences 9, 663-665.

Kennedy A.R., \& Little J.B. (1984) Evidence that a second event in X-ray-induced oncogenic transformation in vitro occurs during cellular proliferation. Rad. Res. 99, $228-248$.

Koshland D.E., Sancar A., Ilanawah P.C., Modrich I. (1994a) DNA repair enzymes and mechanisms. Science 266, 1925-1927; 1954-1960.

Koshland D.E. (1994b) Molecule of the year : the DNA repair enzyme. Science 266, 1925.

Latarjet R. \& Tubiana M. (1989) The risks of induced carcinogenesis after irradiation at small doses. The uncertainties wich remain after the 1988 UNSCEAR report. Int. J. Rad. Oncol. 17, 237-240.

Latarjet R. (1995) Influence du débit de dose (facteur temps) Annexe 3 : Rapport de l'Académie des Sciences sur les faibles doses, Paris.

Le François D., Al Achkar W., Aurias A. Couturier J., Dutrillaux B., Flury-Hérard A., Gerbault-Seureau M., Hoffschir F., Lamoliatte E., Lombard M., Muleris M., Prieur M., Ricoul M., Sabatier L. \& Viegas-Pequignot (1989) Chromosomal aberrations induced by low dose gamma irradiation. Study of R-banded chromosomes of human lymphocytes. Mutation Res. 212, 167-172.

Little J.B. (1989) The relevance of cell transformation to carcinogenesis in vivo. In : Low Dose Radiation, K.F. Baverstock and JW. Stather Eds. p. 396-413, Taylor \& Francis, London.

Little M.P., Hawkins M.M., Shore R.E., Charles M.W. and Hildreth N.G. (1991) Time variations in the risk of cancer following irradiation in childhood. Rad. Res. 136, 304-316.

Lorenz E. (1950) Some biological effects of long continued irradiation. Am. J. Roentgenol. 63, 176. 
Luxin W. et al. (1990) Epidemiological investigation of radiological effects in high background radiation areas of Yangjiang, China. J. Rad. Res. 31, 119-136.

Masse R. (1990) Conséquence de l'exposition aux faibles débits de dose. Apport de l'expérimentaiton animale. Radioprotection 25, 363-383.

Matanoski G.M., Startwell P., Elliott E. et al. (1994) Cancer risks in radiobiologists and radiation workers in Radiation carcinogenesis : epidemiology and biological significance (J. Boice and J. Fraumeni Eds.) Raven Press, New-York.

Matanoski G.M., Sternberg A. \& Elliott E.A. (1987) Does radiation exposure produce a protective effect among radiologists? Health Phys. 52, 637-643.

Meyniel G. (1995) Adaptation - Hormesis : Rapport de Académie des Sciences. Annexe 5, Paris.

Miller A.B., Howe G.R., Sherman G.J., Lindsay J.P., Yaffe M.J., Dinner P.J., Risch H.A. \& Preston D.L. (1989) Mortality from breast cancer after irradiation during fluoroscopic examinations in patients being treated for tuberculosis. New England J. Medicine 321, 1285-1289.

Morlier J.P., Morin M., Chameaud J., Masse R., Bottard S. and Lafuma J. (1992) Importance du rôle du débit de dose sur l'apparition des cancers pulmonaires chez le rat après inhalation de Radon. C.R. Acad. Sc. Paris, 315 (série III), 463-466.

Morin M., Masse R. \& Lafuma J. (1990) Effets cancérogenèses de l'irradiation gamma à faible débit de dose (carcinogenic effects of low dose gamma ray irradiation). C.R. Acad. Sc. Paris 311 (série III), 459-466.

Risk from low-dose exposures - Science Lett. 266, 1141-1145.

Muirhead C.R., Butland B.K., Green B.M. and Draper G.J. (1991) Childhood leukaemia and natural radiation. Lancet 337, 503-504.

Nambi K.S.V., Soman S.D. (1987) Environmental radiation and cancer in India. Health Phys. 52, 635-657.

National Council on Radiation Protection and measurements (1980) Influence of dose and its distribution in time on dose-response relationships for low L.E.T. radiation. NCRP Rep. 64.

Neriishi K., Stram D.O., Vaeth M., Mizanos S. \& Akiba S. (1991) The observed relationships between the occurrence of acute radiation sickness and leukaemia mortality among A-bomb survivors. Rad. Res. 125, 206-213.

Pierce D.A. et Vaeth M. (1989) Cancer risk estimation from the A-bomb survivors : extrapolation to low doses, use of relative risk models and other uncertainties. In : Low dose radiation : biological bases of risk, assessment (Baverstock K.F. and Stather J.W. Eds) Taylor and Francis, London.

Pollycove M. (1995) The issue of the decade : hormesis. Europ. J. Nucl. Med. 22, 399-401.

Raabe O.G. (1984) Comparison of the carcinogenicity of radium and bone-seeking actinides. Health Phys. 46, 1241-1248.

Raabe O.G., Book S.A. \& Parks N.J. (1980) Bone cancer from radium. Canine dose-response explains data for mice and humans. Science 208, 61-64.

Redpath J.L., Sun C., Mendonca M., Colman M., Stanbridge E.J. (1989) The application of a human hybrid cell system to studies of radiation induced cell transformation. In : Cell transformation system relevant to radiation induced cancer in man. (Chadwick et al. Eds) pp. 85-90. Adam Hilger, Bristol.

Rowland R.E., Stehney A.F. (1983) Dose-response relationships for radium-induced bone sarcomas. Health Phys. 44 (suppl 1), 15-31.

Shimizu Y., Kato H., Schull W.J., Preston D., Fujita S. \& Pierce D. (1987) Life Span Study Report 11, part 1, Comparison of risk coefficients for site specific cancer mortality based on the DS86 and T65DR shielded Kerma and organ doses: Technical report, RERF, TR 12-87 (RERF, Hiroshima) p. 61. 
Schneider T., Hubert D., Degrange J.P., Bertin M. (1995) Use of risk projection models for the comparison of mortality from radiation induced breast cancer in various population. Health phys. 68, 452-459.

Stram D.O. (1989) Radiosensitivity or dosimetry error ? Observed relationships between acute and late effets of radiation exposure in the Hiroshima-Nagasaki data - RERF Update (RERF, Hiroshima) p. 5.

Straume T., Egbert S.D., Woolson W.A., Finkel R.C., Kubic P.W., Gove H.E., Sharma P. and Hosni M. (1992) Neutron discrepancies in the DS86 Hiroshima Dosimetry System. Health Phys. 63, 421-426.

Straume T., Harris J., Marchetti A.A. and Egbert S.D. (1992) Neutrons confirmed in Nagasaki and at the army pulsed radiation facility : implications for Hiroshima Dosimetry System. Health Phys. 63, 421-426.

The Science of the Total Environment (1994) 142, Nos 1-2 (radiation exposures in the southern Urals. W. Burkkhart and AM. Kellerer Eds.

Thiessen J.W. (1991) Atomic bomb survivors and low-level radiation. RERF Update 2 (RERF, Hiroshima), p. 4.

Thomson D.T., Mabuchi K., Ron R. et al. (1994) Cancer incidence in atomic bomb survivors - Part II : solid tumors 1958-1987. Radiat. Res. 137, S17-S67.

Thomson J.F. \& Grahn D. (1989) Life shortening in mice exposed to fission neutrons and gamma rays. Exposures to continuous gamma radiation. Radiat. Res. 118, 151-160.

Trott K.R. \& Streffer C. (1990) Radiation exposure and occupation risks. (E. Scherer Ed.) Springer, Berlin pp. 61-74.

Tubiana M., Lafuma J., Masse R., Latarjet R. (1992) The assessment of the carcinogenic efforts of low dose radiation : Brit. Inst. Radiol. report 22, The future of human radiation research pp. 109-118.

Tubiana M., Dutreix J., Wambersie A. \& Bewley D.K. (1990) Introduction to radiobiology, 371 p., Taylor \& Francis, London.

United Nations Scientific Committee on the Effects of Atomic Radiation (UNSCEAR) (1988) Report Sources, Effects and Risks of Ionizing Radiations, 647 pages.

United Nations Scientific Committee on the Effects of Atomic Radiations (UNSCEAR) (1994) Report "Sources and Effects of Ionizing Radiations".

Van Bekkum D.W. \& Bentvelzen (1982) The concept of gene transfer-misrepair mechanisms of radiation carcinogenesis may challenge the linear extrapolation model of risk estimation for low radiation doses, Health Phys. 43, 231-237.

Walinder G. (1995) Has radiation protection become a health hazard ? Karnkraftssakerhet \& Utbildning AB, Stockholm.

Wang J. (1993) Statistical analysis of cancer mortality data of high background radiation areas in Yiangjiang, Chin. J. Rad. Med. Prot. 13, 291-294.

Wang Z., Boice J.D., Wei L. (1990) Thyroid nodularity and chromosome aberrations among women in areas of high background radiation in China. J. Nat. Cancer Inst. 82, 4778-4785.

Weinberg R.A. (1991) Tumor suppressor genes, Science 254, 1138-1146.

Wyngaarden K.L. van, Pauwels F.K.J. Hormesis : are low doses of ionizing radiation harmful or beneficial ?, Eur. J. Nucl. Med. 22, 481-486. 